\title{
Systemic isradipine treatment diminishes calcium-dependent mitochondrial oxidant stress
} \author{
Paul T. Schumacker, ${ }^{2}$ and D. James Surmeier ${ }^{1}$ \\ 'Department of Physiology, and 'Department of Pediatrics, Feinberg School of Medicine, Northwestern University, Chicago, Illinois, USA.
}

Jaime N. Guzman, ${ }^{1}$ Ema Ilijic, ${ }^{1}$ Ben Yang, ${ }^{1}$ Javier Sanchez-Padilla, ${ }^{1}$ David Wokosin, ${ }^{1}$ Dan Galtieri, ${ }^{1}$ Jyothisri Kondapalli,

\begin{abstract}
The ability of the Cav1 channel inhibitor isradipine to slow the loss of substantia nigra pars compacta (SNc) dopaminergic (DA) neurons and the progression of Parkinson's disease (PD) is being tested in a phase 3 human clinical trial. But it is unclear whether and how chronic isradipine treatment will benefit SNc DA neurons in vivo. To pursue this question, isradipine was given systemically to mice at doses that achieved low nanomolar concentrations in plasma, near those achieved in patients. This treatment diminished cytosolic $\mathrm{Ca}^{2+}$ oscillations in SNc DA neurons without altering autonomous spiking or expression of $\mathrm{Ca}^{2+}$ channels, an effect mimicked by selectively knocking down expression of Cav1.3 channel subunits. Treatment also lowered mitochondrial oxidant stress, reduced a high basal rate of mitophagy, and normalized mitochondrial mass demonstrating that Cav1 channels drive mitochondrial oxidant stress and turnover in vivo. Thus, chronic isradipine treatment remodeled SNc DA neurons in a way that should not only diminish their vulnerability to mitochondrial challenges, but to autophagic stress as well.
\end{abstract}

\section{Introduction}

The loss of substantia nigra pars compacta (SNc) dopaminergic (DA) neurons is a defining feature of Parkinson's disease (PD) and is responsible for the core motor symptoms of the disease (1, 2). The selective vulnerability of these neurons has been linked to their distinctive physiology (3). In contrast to most neurons in the brain, SNc DA neurons are slow autonomous pacemakers, spiking at low frequency in the absence of synaptic stimulation $(4,5)$. Somatodendritic $\mathrm{Ca}^{2+}$ entry through Cav1 channels increases the robustness of pacemaking (6-9) and helps meet intracellular bioenergetic needs by stimulating mitochondrial intermediary metabolism and oxidative phosphorylation in a manner similar to that found in muscle (10-14). Although this bioenergetic strategy helps to ensure that ATP levels are sustained at levels sufficient to avoid a suppression of pacemaking activity during periods of stress (13, 15), it increases the generation of mitochondrial reactive oxygen species (ROS) and reactive nitrogen species (RNS) $(13,14)$. Local ROS/RNS generation can damage mitochondrial proteins as well as increase the vulnerability to environmental toxins and genetic mutations associated with PD $(16,17)$. In principle, sustained oxidant stress should drive mitochondrial turnover (mitophagy), creating a proteostatic burden that could synergize with other stressors associated with PD, such as misfolded $\alpha$-synuclein or mutations in glucocerebrosidase (18), to promote neuropathology.

The centrality of Cav1 $\mathrm{Ca}^{2+}$ channels in determining the vulnerability of SNc DA neurons is buttressed by animal studies showing that dihydropyridines (DHPs), which are negative allosteric

Authorship note: JNG, El, and BY contributed equally to this work. Conflict of interest: The authors have declared that no conflict of interest exists. Submitted: June 26, 2017; Accepted: March 13, 2018

Reference information: / Clin Invest. 2018;128(6):2266-2280.

https://doi.org/10.1172/JCI95898. modulators of Cav1 channels, diminish the impact of PD-linked toxins (19-22) and genetic mutations (14). Moreover, epidemiological studies have shown that human use of DHPs is associated with decreased risk and progression of PD (23-28). Based upon these findings, a 5-year, phase 3 clinical trial with the DHP isradipine was initiated by the NIH in 2013 to determine whether it can slow disease progression (ClinicalTrials.gov NCT02168842) (29).

Despite its scientific merits, the clinical trial could fail for several reasons. One is that SNc DA neurons in vivo might compensate for inhibition of Cav1 channels by upregulating their expression or that of other $\mathrm{Ca}^{2+}$ channels with similar properties; indeed, germline deletion of Cav1.3 $\mathrm{Ca}^{2+}$ channels - the channels thought to be largely responsible for sustained $\mathrm{Ca}^{2+}$ entry and mitochondrial stress $(8,20,30)$ - results in upregulation of Cav3 $\mathrm{Ca}^{2+}$ channels in SNc DA neurons and little net change in cytosolic $\mathrm{Ca}^{2+}$ oscillations (31). Another potential stumbling block is that it is not clear that the mitochondrial oxidant stress attributable to $\mathrm{Ca}^{2+}$ entry through Cav1 channels is sufficient to actually damage mitochondria in otherwise healthy SNc DA neurons in vivo. Although there is indirect evidence to support this proposition (3), there is no direct demonstration that this is the case. A third caveat, which has recently been argued (32), is that even if $\mathrm{Ca}^{2+}$ entry through Cav1 channels triggers mitochondrial damage in vivo, it is not evident that a sufficient level of channel inhibition can be achieved with systemic drug administration to diminish it.

To address these questions, a combination of electrophysiological, optical, and genetic approaches was used to study SNc DA neurons in healthy mice after they had been chronically administered isradipine with subcutaneous osmotic minipumps. The overall goal was to determine whether clinically relevant doses of isradipine effectively engaged Cav1 $\mathrm{Ca}^{2+}$ channels in brain SNc DA neurons in vivo and, if so, what the consequences of this engagement were. Optical tools were used to quantitatively measure 
A

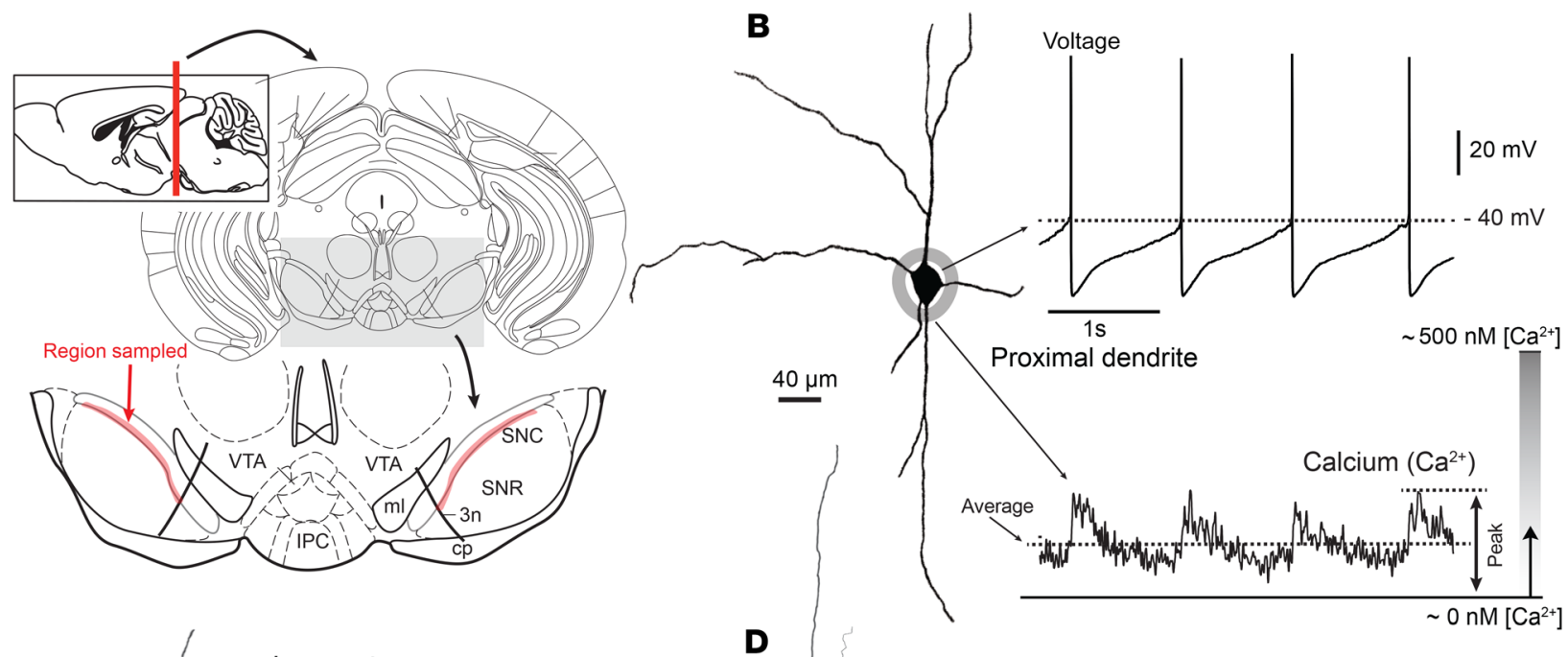

C

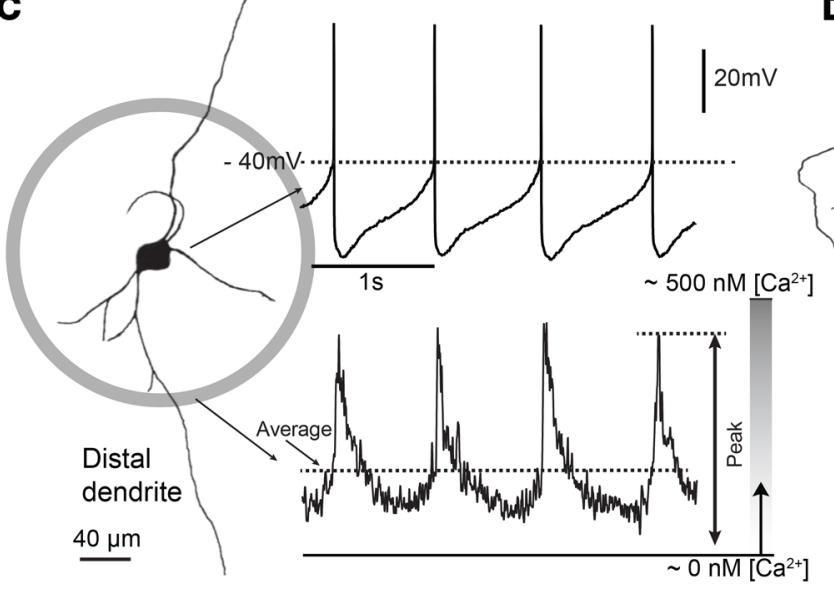

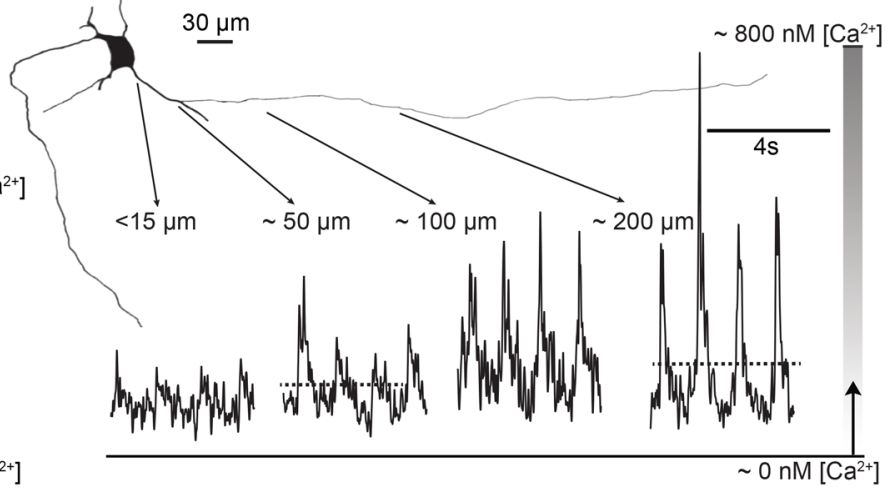

Figure 1. Combined patch clamp and Fura-2 $\mathrm{Ca}^{2+}$ imaging from SNc DA neurons revealed large oscillations in cytosolic [C $\left.\mathrm{a}^{2+}\right]$ during pacemaking. (A) Schematic coronal section of the midbrain, positioned $3.5 \mathrm{~mm}$ posterior to bregma. At the bottom, magnified ventral part of the midbrain showing sampled region of SNc (highlighted in red). (B) Whole-cell recording from a SNc DA neuron shown at left as a representative reconstruction of a Fura-2 -filled cell. At the bottom, 2PLSM measurement of Fura-2 fluorescence at a proximal dendritic location is shown ( 15 $\mu \mathrm{m}$ from the soma). (C) Somatic recording during imaging at a distal dendritic location ( $100 \mu \mathrm{m}$ from the soma from the projection image of a SNc DA neuron). Note the increase in Ca ${ }^{2+}$ transient at the distal imaging site. (D) $\mathrm{Ca}^{2+}$ transients increased along the dendrite of shown SNc DA neuron ranging from 15 to $200 \mu \mathrm{m}$ from the soma.

intracellular $\mathrm{Ca}^{2+}$ concentrations as well as mitochondrial oxidant stress, turnover, and mass. These studies not only support the proposition that sustained inhibition of Cav1 channels is achievable in vivo without inducing maladaptive compensations, they also provide the first clear demonstration, to our knowledge, that Cav1 channel-driven mitochondrial oxidant stress in vivo is sufficient to accelerate mitophagy in SNc DA neurons and to decrease mitochondrial mass. In addition to strengthening the rationale for the ongoing clinical trial, these studies also establish a clear mechanistic connection between mitochondrial and proteostatic theories of PD pathogenesis.

\section{Results}

Pacemaking was accompanied by large fluctuations in dendritic $\left[\mathrm{Ca}^{2+}\right]$. To generate a quantitative estimate of intracellular $\mathrm{Ca}^{2+}$ concentration $\left(\left[\mathrm{Ca}^{2+}\right]\right)$, SNc DA neurons in ex vivo brain slices from young mice (P25-P35) (Figure 1A) were patched in wholecell mode with an electrode filled with Fura-2 $(100 \mu \mathrm{M})$ and then imaged using 2-photon laser-scanning microscopy (2PLSM). The imaging protocol took advantage of the fact that, with long wavelength excitation $(780 \mathrm{~nm})$, the emission of Fura-2 falls with rising $\left[\mathrm{Ca}^{2+}\right]$ (33). This allowed the maximum fluorescence (which is proportional to dye concentration) to be determined by hyperpolarizing neurons with brief current steps injected through the patch electrode (to pull $\left[\mathrm{Ca}^{2+}\right]$ low). At each imaging site, this was done approximately 20 seconds prior to acquiring fluorescence measurements associated with normal pacemaking, allowing a quantitative estimate of intracellular $\left[\mathrm{Ca}^{2+}\right]$ to be calculated $(34,35)$ (see Methods and Supplemental Figures 1 and 2; supplemental material available online with this article; https://doi. org/10.1172/JCI95898DS1). At near physiological temperature $\left(30-32^{\circ} \mathrm{C}\right), 20$ to 30 minutes after beginning Fura-2 dialysis, the kinetics and amplitude of spiking-associated fluorescence transients had stabilized at distal $(\sim 100 \mu \mathrm{m})$ dendritic sites (Supplemental Figure 2, A-E), indicating that the dye concentration was near equilibrium. Although Fura-2 buffering of free $\mathrm{Ca}^{2+}$ will cause estimates of transient changes in intracellular $\left[\mathrm{Ca}^{2+}\right]$ to be slower and smaller than they would be in the absence of dye, in 

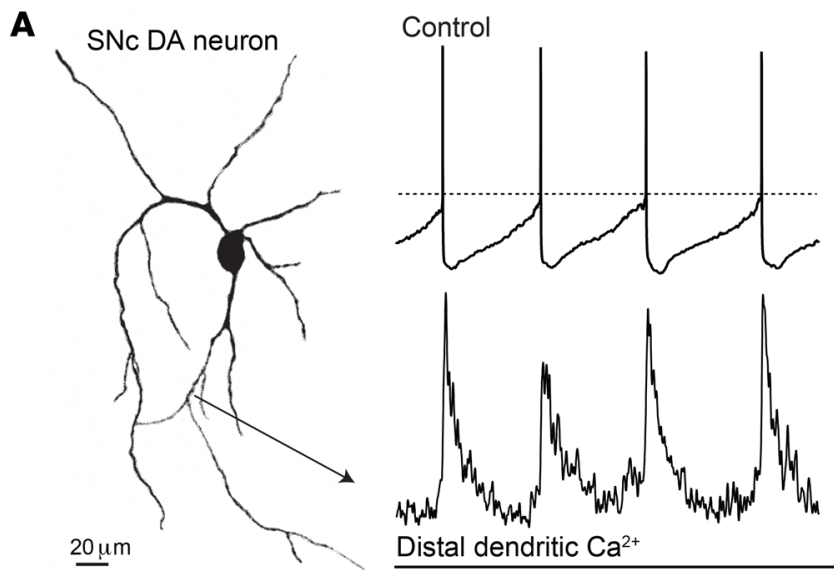

B
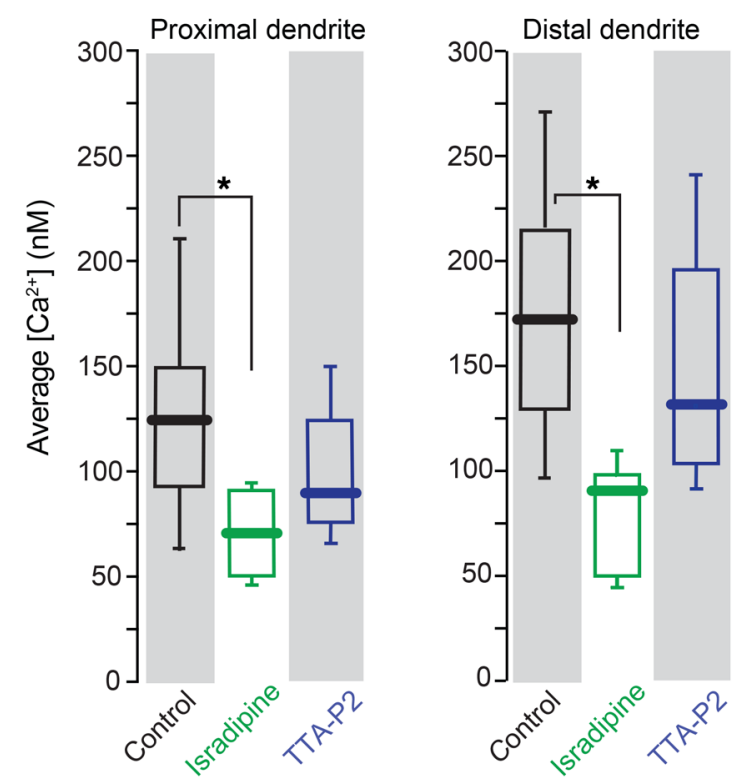
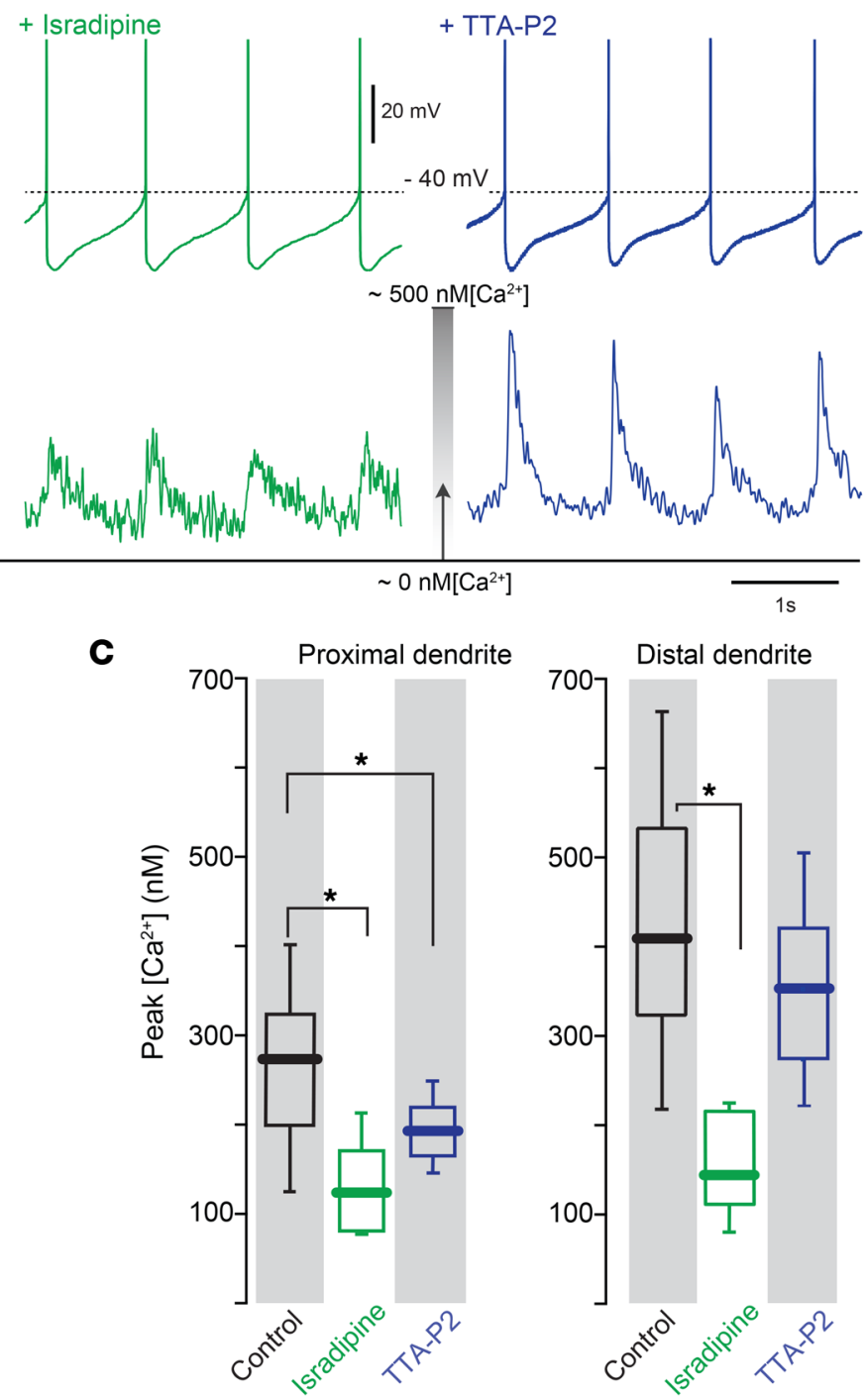

Figure 2. Both Cav1 and $\mathrm{Cav3} \mathrm{Ca}^{2+}$ channels contributed to dendritic $\mathrm{Ca}^{2+}$ oscillations in SNc DA neurons. (A) Projection image of a SNc DA neuron recorded from with a somatic patch electrode containing Fura-2 showing $\mathrm{Ca}^{2+}$ transient in distal dendrite. From left, control condition (black trace), with bath incubation of either $1 \mu \mathrm{M}$ isradipine (green trace) or $1 \mu \mathrm{M}$ TTA-P2 (blue trace). Note diminished dendritic $\mathrm{Ca}^{2+}$ transient with $1 \mu \mathrm{M}$ isradipine. (B and C) Box plots summarizing the average and the peak $\left[\mathrm{Ca}^{2+}\right]$, respectively, of the control, isradipine, and TTA-P2 treatment for both proximal and distal dendrites. Sample sizes for proximal dendrites were as follows: control group, $n=28$ neurons from 23 mice; isradipine-treated group, $n=6$ neurons from 4 mice; and TTA-P2-treated group, $n=9$ neurons from 7 mice. In distal dendrites, sample sizes were as follows: control group, $n=31$ neurons from 22 mice; isradipinetreated group, $n=7$ neurons from 6 mice; and TTA-P2-treated group, $n=11$ neurons from 7 mice. Data were analyzed using 1-tailed Mann-Whitney $U$ test with Dunn's correction for multiple comparisons. ${ }^{*} P<0.05$.

these near steady-state conditions, peak and average $\left[\mathrm{Ca}^{2+}\right]$ estimates allow comparisons to be drawn between different regions of a neuron and across neurons.

Because dendrites appear to be particularly vulnerable to aging and PD $(20,36,37)$, SNc DA neurons were imaged at both proximal ( $10 \mu \mathrm{m}$ from the soma) and distal $(\sim 100 \mu \mathrm{m}$ from soma) dendritic locations. During pacemaking, $\left[\mathrm{Ca}^{2+}\right]$ rose and fell at both locations, as expected from previous work $(7,38)$. The average and peak $\left[\mathrm{Ca}^{2+}\right]$ estimates (generated by averaging line scans over more than 4 successive pacemaking cycles) exhibited a strong proximal-to-distal gradient, rising with distance from the soma. With slow pacemaking $(\sim 1 \mathrm{~Hz})$, in proximal dendrites, the spike-associated $\left[\mathrm{Ca}^{2+}\right]$ estimate typically rose to a peak near $200 \mathrm{nM}\left[\mathrm{Ca}^{2+}\right]$ (Figure 1B). In distal dendrites about $100 \mu \mathrm{m}$ from the soma, the spike-associated peak $\left[\mathrm{Ca}^{2+}\right]$ estimates were commonly twice as large $(\sim 400 \mathrm{nM})$ (Figure $1 \mathrm{C})$. This gradient can be seen more clearly in a parfocal dendrite where line scans were performed at both proximal and distal locations in the same cell (Figure 1D). Across the sampled population of SNc DA neurons, the peak and average $\left[\mathrm{Ca}^{2+}\right]$ estimates were significantly larger in distal dendrites than in proximal dendrites, as were transient area measurements (Supplemental Figure 2F). Peak $\left[\mathrm{Ca}^{2+}\right]$ estimates increased with spiking frequency in both the proximal and distal dendrites (Supplemental Figure 3). To determine whether the peak $\left[\mathrm{Ca}^{2+}\right]$ was being underestimated because of dye saturation, experiments were performed with a lower affinity dye (BisFura); these estimates were not significantly different from those with Fura-2 (Supplemental Figure 4). 
A

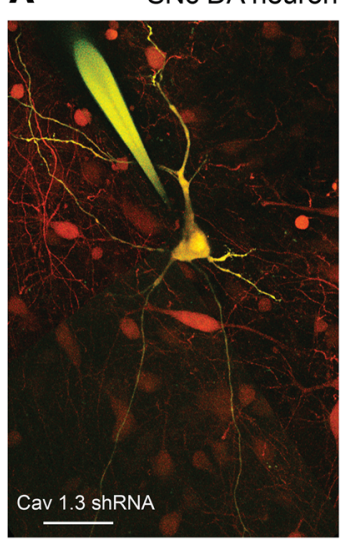

B

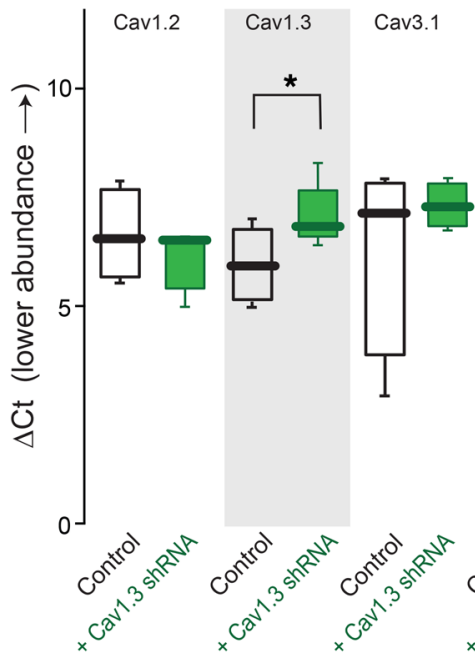

C

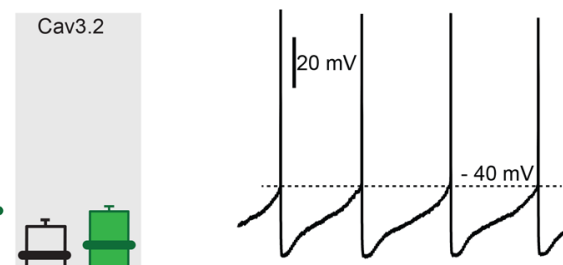

+ Cav1.3 shRNA

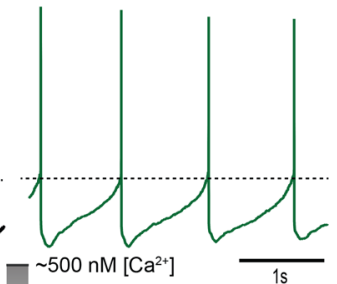

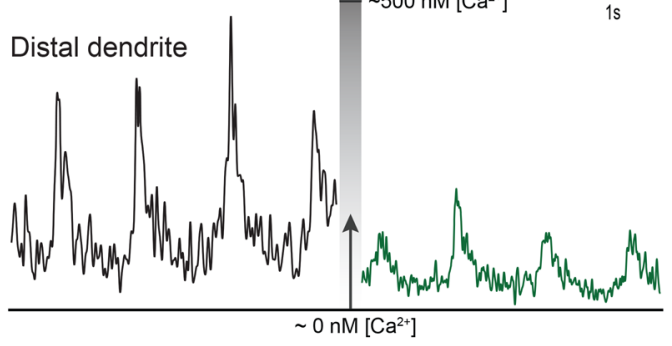

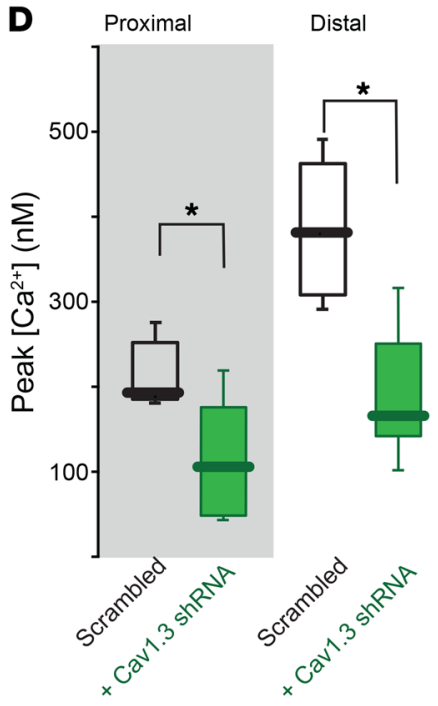

E Somatic voltage

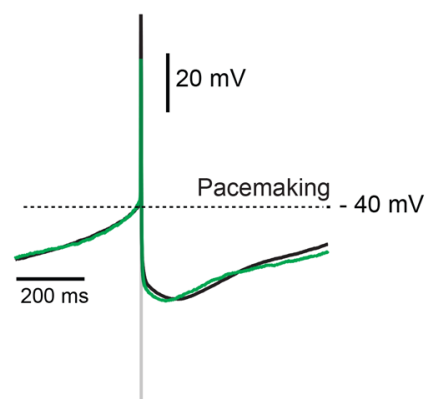

$\mathbf{F}$
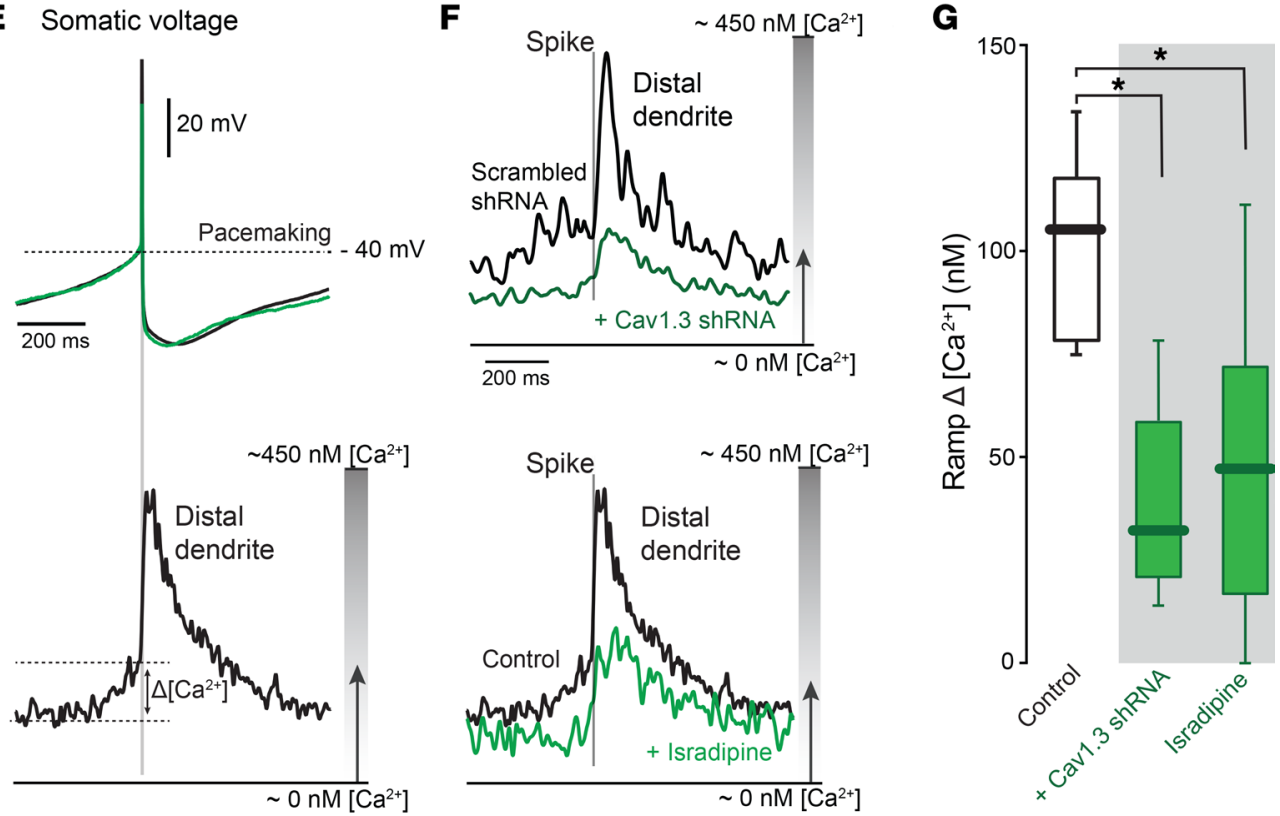

Figure 3. Selective knockdown of Cav1.3 mRNA diminished both proximal and distal Ca ${ }^{2+}$ oscillations in SNc DA neurons. (A) Cav1.3 shRNA-infected SNc DA neurons (red) and patched neurons filled with Fura-2 dye (yellow). Scale bar: $40 \mu \mathrm{m}$. (B) Cav1.3 knockdown decreased Cav1.3 mRNA, but didn't change Cav1.2, Cav3.1, and Cav3.2 mRNA ( $n=4$ tissue samples from 3 mice for Cav1.2, Cav3.1, and Cav3.2; $n=5$ tissue samples from 3 mice for Cav1.3). (C) Wholecell recording from a SNc DA neuron with scrambled shRNA (black trace) and Cav1.3 shRNA (green trace). At the bottom, Ca ${ }^{2+}$ transients in distal dendrites were diminished with Cav1.3 shRNA but not scrambled shRNA. (D) Summary of peak [Ca $\left.{ }^{2+}\right]$ in proximal and distal dendrites of experiments as in C. (Proximal dendritic locations: scrambled shRNA, $n=5$ neurons from 4 mice; Cav1.3 shRNA, $n=7$ neurons from 5 mice; distal dendrites, scrambled shRNA, $n=8$ neurons from 4 mice; Cav1.3 shRNA, $n=8$ neurons from 6 mice). (E) Upper panel shows average spike trajectory; lower panel shows average Ca ${ }^{2+}$ transients in the distal dendrite. (F) Upper panel shows average $\mathrm{Ca}^{2+}$ transients with injected scrambled shRNA (black trace) or Cav1.3 shRNA (green trace) in distal dendrites; lower panel shows average distal dendritic $\mathrm{Ca}^{2+}$ transients in the presence of $1 \mu \mathrm{M}$ isradipine (green trace) and control (black trace). (C) Box plots summarizing the ramp [ $\left.\mathrm{Ca}^{2+}\right]$ in control ( $n=8$ neurons from 6 mice), Cav1.3 shRNA-injected ( $n=5$ neurons from 5 mice), and $1 \mu \mathrm{M}$ isradipine-treated ( $n=8$ neurons from 4 mice) SNc DA neurons. Note that Cav1.3 shRNA and isradipine suppressed slow increase in [Ca ${ }^{2+}$ that preceded the spike. Data were analyzed using 1-tailed Mann-Whitney $U$ test with Dunn's correction for multiple comparisons. ${ }^{*} P<0.05$.

To create a developmental and cellular context, 2 other experiments were conducted. First, SNc DA neurons from very young postnatal mice (P5-P10) were examined. Both the average and peak cytosolic $\left[\mathrm{Ca}^{2+}\right]$ estimates during pacemaking were significantly lower in these neurons than in older, more mature neurons (Supplemental Figure 5). Second, ventral tegmental area (VTA) DA neurons, which are less vulnerable than SNc DA neurons (3), were studied. Although VTA DA neurons have pacemaking properties that are similar to those of SNc DA neurons (39), cytosolic $\left[\mathrm{Ca}^{2+}\right]$ during pacemaking was significantly lower than that in SNc DA neurons, regardless of dendritic location (Supplemental Figure 6).

Cav1 and Cav3 $\mathrm{Ca}^{2+}$ channels contributed to dendritic $\mathrm{Ca}^{2+}$ oscillations. Previous work has implicated both Cav1 and Cav3 $\mathrm{Ca}^{2+}$ 
A

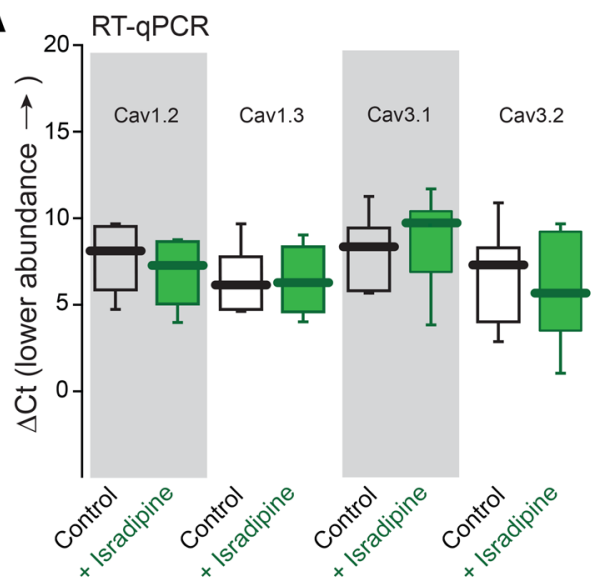

B

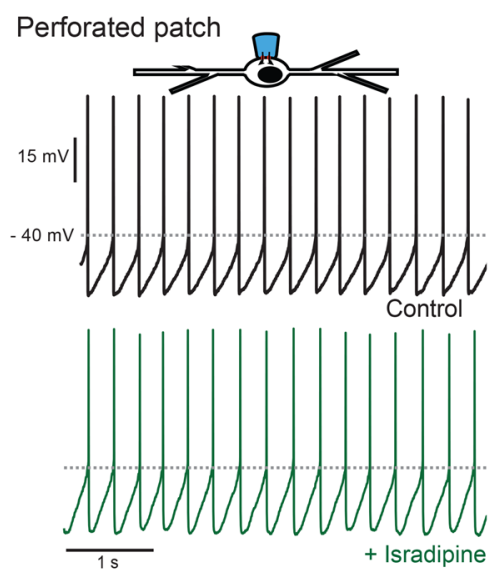

C

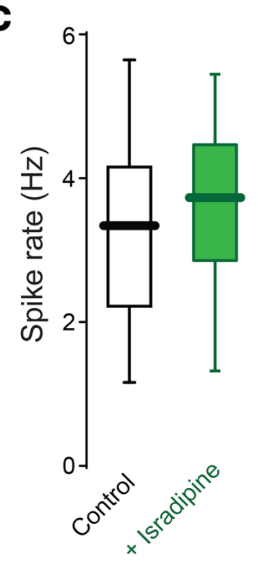

D SNc DA neuron

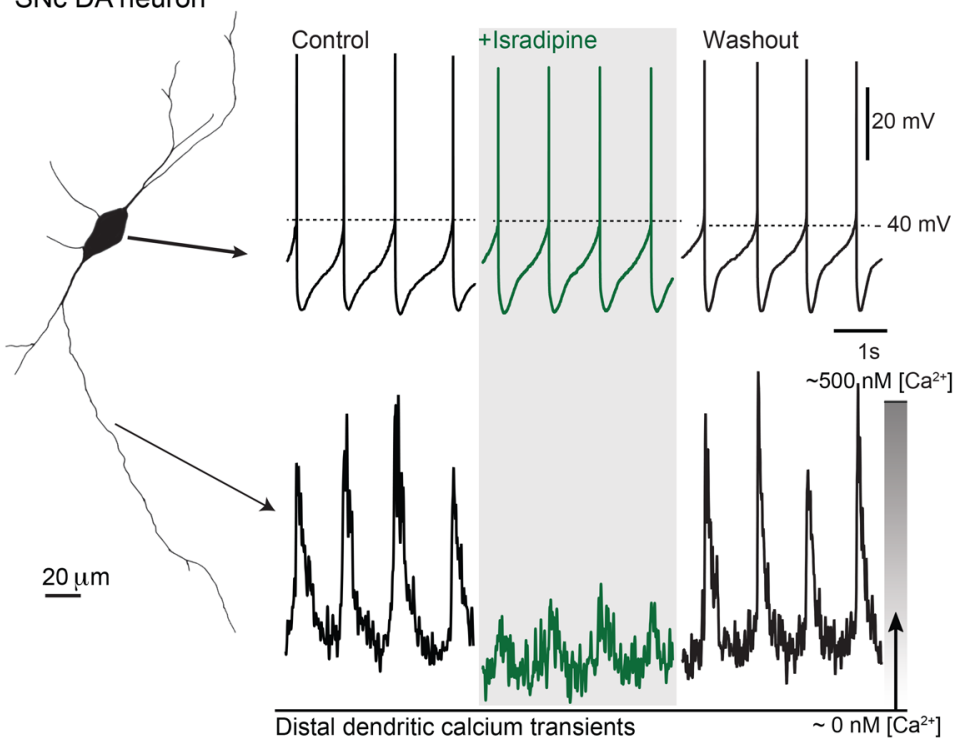

E Proximal dendrites

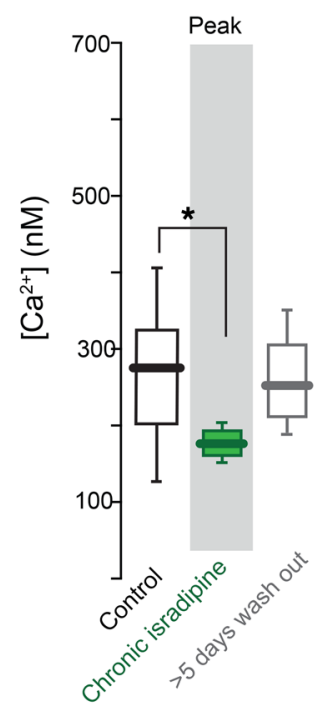

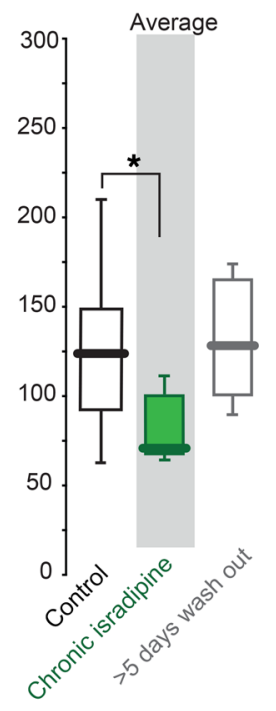

F Distal dendrites
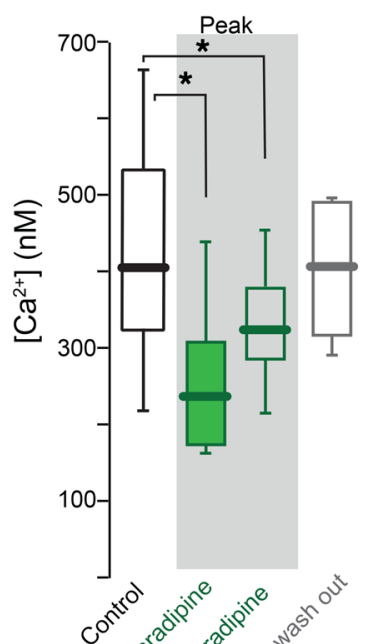

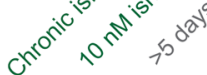

G

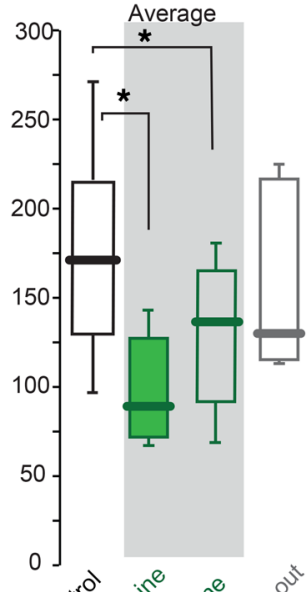

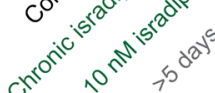

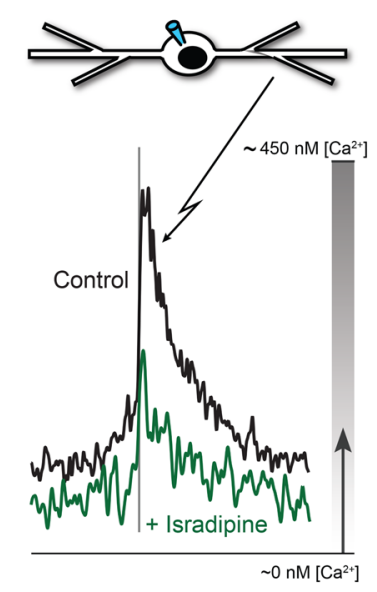

H

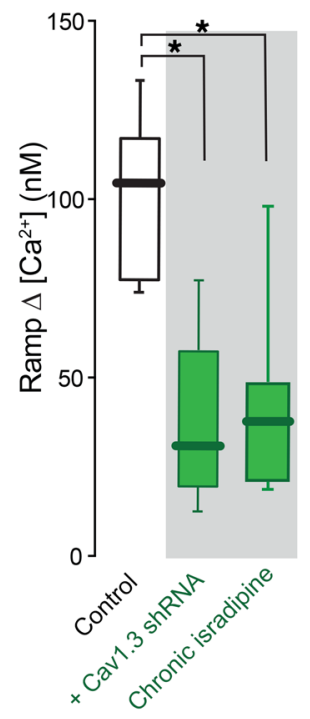


Figure 4. Chronic administration of isradipine reduced dendritic $\mathrm{Ca}^{2+}$ oscillations without inducing compensations in channel expression. (A) qPCR revealed no significant change in the mRNA expression of Cav1.3, Cav1.2, Cav3.1, and Cav3.2 $\mathrm{Ca}^{2+}$ channels after chronic isradipine treatment $(n=6$ tissues from each group os 3 mice for Cav1.2 and Cav1.3; $n=7$ tissues from each group of 3 mice for Cav3.1 and Cav3.2). (B) Perforated-patched recordings from vehicle-treated (black) and isradipine-treated (green) neurons. (C) Pacemaking rates in SNc DA neurons from control and isradipine-treated mice were unchanged (vehicle, 9 neurons from 4 mice; isradipine, 11 neurons from 4 mice). (D) Whole-cell somatic recording (left) and distal dendritic $\mathrm{Ca}^{2+}$ transients; chronic isradipine treatment (green trace, upper middle) reduced dendritic $\mathrm{Ca}^{2+}$ oscillations. Removal of the pumps (washout) led to restoration of the oscillation (lower right, black traces). (E) Peak and average proximal dendritic $\mathrm{Ca}^{2+}$ measurements under control (from Figure 2, B and $\mathrm{C}$ ), isradipine-treated, and isradipine-washout conditions (control, 28 neurons from 23 mice; chronic isradipine, 5 neurons from 5 mice; washout, 5 neurons from 5 mice). (F) Summary of data from distal dendrites (control, 31 neurons from 22 mice; chronic isradipine, 8 neurons from 8 mice; acute $10 \mathrm{nM}$ isradipine, 14 neurons from 6 mice; washout, 5 neurons from 5 mice). (C) Average $\mathrm{Ca}^{2+}$ transients in control (black trace) and isradipine-treated (green trace) distal dendrite of an SNc DA neuron. (H) Box plots summarizing the ramp $\left[\mathrm{Ca}^{2+}\right]$ in control ( $n=8$ neurons from 6 mice, from Figure 3G), Cav1.3 shRNA-injected ( $n=5$ neurons from 5 mice, from historical Cav1.3 shRNA in Figure 3G), and chronic isradipine ( $n=9$ neurons from 8 mice) SNc DA neurons. Data were analyzed using 1-tailed Mann-Whitney $U$ test with Dunn's correction for multiple comparisons. ${ }^{*} P<0.05$.

channels in the dendritic $\mathrm{Ca}^{2+}$ oscillations in SNc DA neurons (7, 40 ). However, none of this work has used quantitative $\mathrm{Ca}^{2+}$ imaging to estimate the relative contribution of each channel type to oscillations at defined subcellular locations. To estimate the contribution of Cav1 channels to dendritic $\left[\mathrm{Ca}^{2+}\right]$ oscillations, isradipine was bath applied at a saturating concentration $(1 \mu \mathrm{M})$ that is known to inhibit Cav1 channels, but not to disrupt the gating of other plasma membrane channels (7). Isradipine at this concentration had no effect on the pacemaking rate $(7,41)$. Nevertheless, it reduced the average and peak $\left[\mathrm{Ca}^{2+}\right]$ by about half in both proximal and distal dendrites (Figure 2, $\mathrm{A}-\mathrm{C}$ ).

To estimate the contribution of $\mathrm{Cav} 3$ channels to dendritic $\mathrm{Ca}^{2+}$ transients during pacemaking, the specific Cav3 channel blocker TTA-P2 $(1 \mu \mathrm{M})$ was used $(42,43)$. At proximal dendritic sites, bath application of TTA-P2 significantly reduced the peak $\left[\mathrm{Ca}^{2+}\right]$ during pacemaking; however, the average $\left[\mathrm{Ca}^{2+}\right]$ in proximal dendrites was not significantly diminished by TTA-P2 (although the data trended in this direction) (Figure 2, A-C). TTA-P2 had no effect on the distal dendritic $\left[\mathrm{Ca}^{2+}\right]$ oscillation (Figure 2, A-C). Thus, Cav3 channels appear to be primarily located in the proximal part of the dendritic tree of SNc DA neurons and make only a modest contribution to the oscillations in $\left[\mathrm{Ca}^{2+}\right]$ during pacemaking.

Two types of Cav1 channel - one with a pore-forming Cav1.2 subunit and the other with a Cav1.3 subunit - are expressed by SNc DA neurons (41). The low-threshold Cav1.3 $\mathrm{Ca}^{2+}$ channels have been postulated as helping to drive the dendritic membrane potential to spike threshold during pacemaking (7-9), but this has been difficult to prove because there are no widely accepted selective inhibitors of Cav1.3 channels $(44,45)$ and germline deletion of the gene coding for Cav1.3 (Cacna1d) leads to compensatory upregulation in Cav3 channel expression (31). Hence, to determine the role of Cav1.3 channels, a genetic knockdown approach was used. An shRNA targeting Cav1.3 mRNA that had previously been shown to be selec- tive and potent (46) was validated in heterologous expression systems; this construct (and a scrambled control) were packaged into an adenoassociated virus (AAV) vector and then stereotaxically injected into the SNc of P21 mice (Figure 3A). Reverse-transcription quantitative PCR (RT-qPCR) analysis of the SNc taken 7 to 10 days later confirmed the specificity of the knockdown as well as its potency (Figure 3B); importantly, viral Cav1.3 knockdown did not result in upregulation of Cav1.2 or Cav3 channel mRNAs (Figure 3B). The scrambled shRNA had no effect on cytosolic $\left[\mathrm{Ca}^{2+}\right]$ (Figure 3C; statistical comparison with data in Figure 2, B and C; $P>0.05$, Mann-Whitney $U$ test). However, in SNc DA neurons expressing the Cav1.3 shRNA, the $\left[\mathrm{Ca}^{2+}\right]$ in both proximal and distal dendrites was significantly reduced (Figure 3, C and D).

A key feature of Cav1.3 channels is their activation at membrane potentials reached before the spike. Indeed, in distal dendrites, the $\left[\mathrm{Ca}^{2+}\right]$ began to rise before the spike. This was seen most clearly by averaging the $\left[\mathrm{Ca}^{2+}\right]$ signal over successive spikes, using the somatic spike as the centering point (Figure 3E); the difference between the $\left[\mathrm{Ca}^{2+}\right]$ estimate at the trough of the oscillation and that just before the spike was used to estimate the magnitude of this ramp in $\left[\mathrm{Ca}^{2+}\right]$ (Figure 3E). Attesting to its role in this phenomenon, Cav1.3 knockdown significantly diminished the change in $\left[\mathrm{Ca}^{2+}\right]$ preceding the spike as well as the transient associated with the spike (Figure 3, F and $\mathrm{G}$ ). Reanalyzing the data in Figure 2 revealed that isradipine (a nonspecific inhibitor of Cav1.3 channels) also significantly reduced the ramp $\left[\mathrm{Ca}^{2+}\right]$ (Figure 3, F and G). Another characteristic of the dendritic $\mathrm{Ca}^{2+}$ oscillation that could be traced to subthreshold activation of Cav1.3 channels was its relative insensitivity to the failure of the somatic spike (Supplemental Figure 7). Taken together, these results provide strong evidence that Cav1.3 $\mathrm{Ca}^{2+}$ channels are important determinants of the dendritic oscillations in $\left[\mathrm{Ca}^{2+}\right]$.

Chronic isradipine treatment reduced somatodendritic $\mathrm{Ca}^{2+} \mathrm{lev}$ els. To chronically administer isradipine, mice were implanted with subcutaneous osmotic minipumps that delivered $3 \mu \mathrm{g} / \mathrm{g}$ body weight/d. This dose has previously been shown to protect SNc DA neurons from modest (but not high) doses of the toxins 6-hydroxydopamine (6-OHDA) and 1-methyl-4-phenyl-1,2,3,6-tetrahydropyridine (MPTP) $(19,20)$, like other DHPs (21). After 7 to 10 days of isradipine treatment, the open-field behavior of isradipinetreated mice was indistinguishable from that of controls, arguing that this dose did not induce any gross alterations in the activity of SNc DA neurons in vivo (Supplemental Figure 8). Plasma concentration of isradipine achieved by this regimen was estimated to be around $5 \mathrm{nM}$ (Supplemental Figure 9). This estimate is lower, but close to that of 2 previous rodent studies $(32,47)$; it is also within the low nanomolar range expected to be achieved in humans with oral administration of either immediate release or controlled release format isradipine $(48,49)$ (Supplemental Figure 9).

At this point, mice were sacrificed either for analysis of $\mathrm{Ca}^{2+}$ channel subunit expression or for electrophysiology and $\mathrm{Ca}^{2+}$ imaging. Systemic isradipine treatment did not change nigral expression of mRNA coding for the pore-forming subunits of Cav1.2, or Cav1.3 channels (Figure 4A); moreover, expression of mRNA for the pore-forming subunits of Cav3.1 and Cav3.2 channels, the 2 primary Cav3 (or T-type) $\mathrm{Ca}^{2+}$ channels in SNc DA neurons (31), also was unchanged (Figure 4A). In ex vivo slices from chronically treated mice, there was no discernible change in the pacemaking 
A
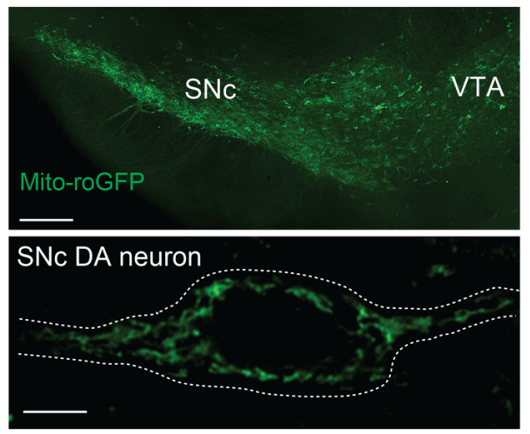

D
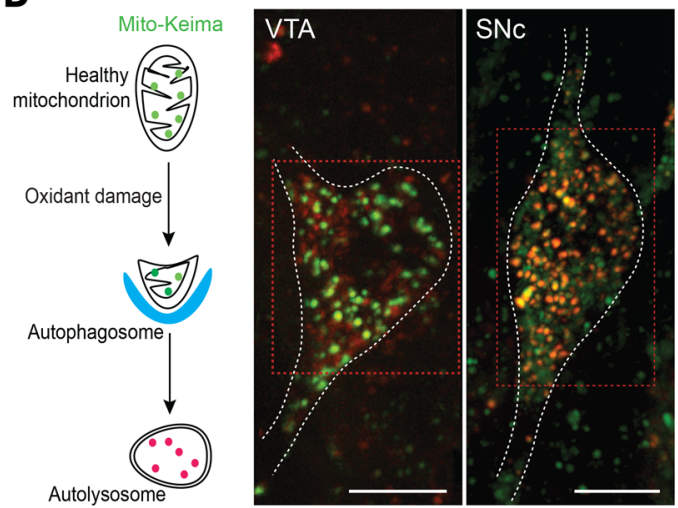

B

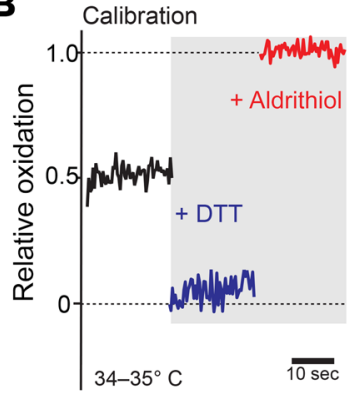

$\mathbf{E}$

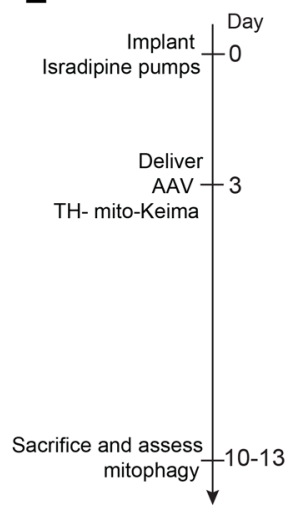

C
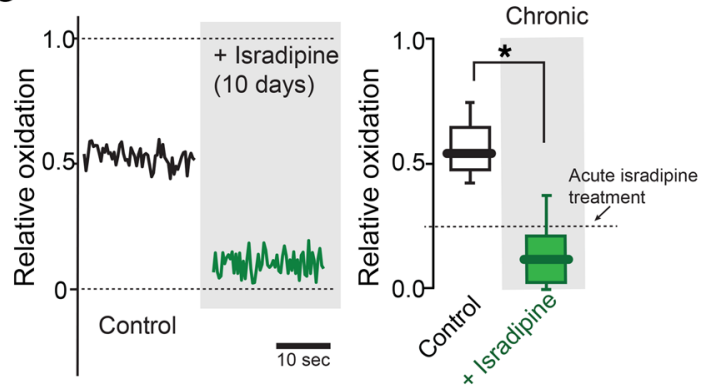

F

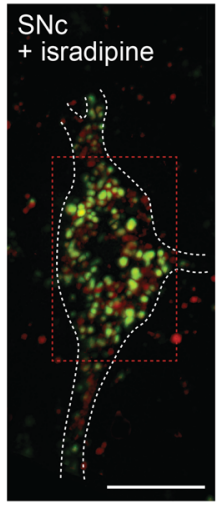

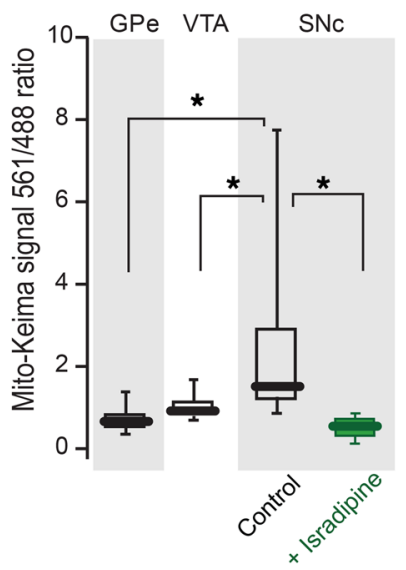

Figure 5. Chronic isradipine treatment decreased mitochondrial oxidant stress and mitochondrial turnover, resulting in increased mitochondrial density. (A) Top: low-magnification image of a midbrain slice from transgenic TH-mito-roGFP mouse showing fluorescence in SNc and VTA. Bottom: higher-magnification image of a SNc DA neuron showing mitochondrial labeling, with a white dashed line at cell membrane. (B) Mito-roGFP measurements from a SNc DA neuron before (control, black trace) and after application of dithiothreitol (DTT, blue trace) and aldrithiol (red trace). (C) Left: MitoroGFP measurements in SNc DA neuron after chronic systemic administration of isradipine showing diminished mitochondrial oxidation. Right: box plots summarizing median redox measurements in control (7 neurons from 4 mice) and isradipine-treated mice (8 neurons from 4 mice). Dashed line indicates published data (14). (D) Left: drawing showing the $\mathrm{pH}$-dependent fluorescent properties of mito-Keima, which allowed rapid determination as to whether the protein was in the mitochondria ( $\mathrm{pH} 8.0$ ) or the lysosome ( $\mathrm{pH}$ 4.5). Mito-Keima fluorescence signal from $488 \mathrm{~nm}$ laser excitation (neutral pH) is shown in green and the signal from $561 \mathrm{~nm}$ laser excitation (acidic) is shown in red. Note the difference in mito-Keima fluorescence between VTA and SNc DA neuron. (E) Left: schematic presentation of chronic isradipine treatment and mito-Keima experiments. Right: chronic isradipine treatment changed the ratio of mito-Keima in SNc DA neurons compared with control. (F) Box plots summarizing the rate of mitophagy in GPe (10 neurons from 4 mice), VTA DA neurons ( 15 neurons from 6 mice), and control (21 neurons from 8 mice) and isradipine-treated SNc DA neurons (40 neurons from 7 mice). Note that isradipine significantly decreased the rate of mitophagy in SNc DA neurons. Data were analyzed using 1-tailed Mann-Whitney $U$ test with Dunn's correction for multiple comparisons. ${ }^{*} P<0.05$. Scale bars: $10 \mu \mathrm{m}$ (A, top); $5 \mu \mathrm{m}$ (A, bottom); $10 \mu \mathrm{m}$ (D, E).

rate of SNc DA neurons recorded in perforated patch mode (Figure $4, \mathrm{~B}$ and $\mathrm{C})$. However, the average and peak dendritic $\left[\mathrm{Ca}^{2+}\right]$ during pacemaking was significantly diminished (Figure 4, D-F). In proximal dendrites, the oscillation in $\left[\mathrm{Ca}^{2+}\right]$ was about a third lower that seen in untreated cells (Figure 4E). In distal dendrites, the reduction in $\left[\mathrm{Ca}^{2+}\right]$ was close to half (Figure $4 \mathrm{~F}$ ). Moreover, as expected from acute inhibition of Cav1.3 channels, the slow ramp in $\left[\mathrm{Ca}^{2+}\right]$ prior to the spike was attenuated by chronic treatment (Figure 4, $\mathrm{G}$ and $\mathrm{H}$ ).

One complication in these experiments was that with removal of the brain and slicing, the concentration of isradipine in the extracellular space was inescapably altered. Initially, the artificial cerebrospinal fluid bathing the cells included $10 \mathrm{nM}$ isradipine to slow washout. But omission of isradipine altogether had no significant effect on measured $\mathrm{Ca}^{2+}$ transients within 3 to 4 hours of sacrifice (Supplemental Figure 10). Given that isradipine is lipophilic and strongly partitions into the lipid membrane (50), the slow reversal in the effects of chronic exposure was not surprising. To determine whether the effects of isradipine reversed over a longer period of time, pumps were removed from chronically treated mice and then the mice were sacrificed 5 to 7 days later. SNc DA neurons from these mice had normal cytosolic oscillations in $\left[\mathrm{Ca}^{2+}\right]$ (Figure 4, D-F). Thus, the effects of isradipine treatment on cytosolic $\left[\mathrm{Ca}^{2+}\right]$ were reversible on the time scale of days.

To determine whether the effects of chronic isradipine treatment could be reproduced by acute treatment at a concentration near that achieved in vivo, slices from untreated mice were incubated with $10 \mathrm{nM}$ isradipine for 60 minutes and then SNc DA neurons were patched and imaged. Isradipine exposure at this concentration significantly diminished peak and average dendritic $\left[\mathrm{Ca}^{2+}\right]$, as did chronic, systemic treatment with isradipine (Figure $4 \mathrm{~F}$ and Supplemental Figure 11). As expected from the voltage dependence of the inhibition of Cav1 channels by DHPs (51), the magnitude of the inhibition by isradipine increased with spike rate (Supplemental Figure 11), suggesting that in vivo, where spiking 

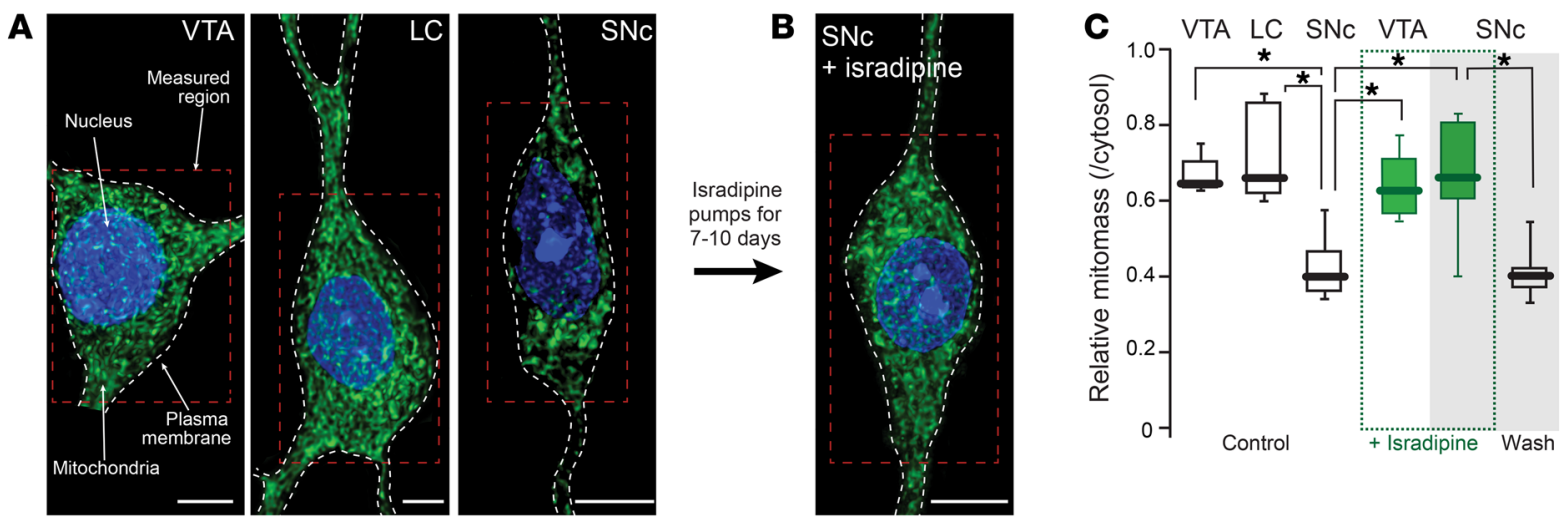

Figure 6. Mitochondrial mass in SNc DA neurons rises after chronic isradipine treatment. (A) Mitochondria in VTA DA, LC, and SNc DA neurons shown with mito-roGFP fluorescence. (B) Note the increased mitochondrial mass in SNc DA neurons after chronic isradipine treatment. (C) Relative mitochondrial mass in cytosol of VTA DA ( 5 neurons from 4 mice) and of LC neurons ( 5 neurons from 4 mice) was significantly higher than that in SNc DA (8 neurons from 5 mice) neurons. Isradipine did not change mitochondrial mass in VTA DA neurons (5 neurons from 4 mice), but did significantly increase it in SNc DA neurons (6 neurons from 5 mice). Removal of isradipine for 4 weeks (washout) showed return of mitochondrial mass to low, control values in SNc DA neurons (8 neurons from 6 mice). Scale bars: $10 \mu \mathrm{m}$ (A); $8 \mu \mathrm{m}$ (B). Data were analyzed using 1-tailed Mann-Whitney $U$ test with Dunn's correction for multiple comparisons. ${ }^{*} P<0.05$.

rates are accelerated by excitatory synaptic input, the magnitude of the Cav1 channel inhibition should be even larger.

Chronic treatment decreased oxidant stress and mitophagy. $\mathrm{Ca}^{2+}$ entry through Cav1 channels increases mitochondrial oxidant stress in SNc DA neurons (14). Several lines of evidence suggest that this is attributable to the ability of $\mathrm{Ca}^{2+}$ currents through Cav1 channels to trigger mitochondrial $\mathrm{Ca}^{2+}$ influx, which then stimulates oxidative phosphorylation (52). As chronic isradipine treatment diminished cytosolic $\mathrm{Ca}^{2+}$ oscillations, it was expected to lower mitochondrial oxidant stress. Indeed, in SNc DA neurons from systemically treated mice, mitochondrial matrix oxidant stress measured with the genetically encoded mitochondrially targeted redox-sensitive GFP (mito-roGFP) probe expressed under control of the tyrosine hydroxylase promoter (Figure 5, A and B) was significantly lower $(P<0.05)$. In fact, in neurons from chronically treated mice, mitochondrial oxidant stress was as low as that achieved by acute inhibition of Cav1 channels with $1 \mu \mathrm{M}$ isradipine (Figure 5C).

Chronic oxidant stress should, in principle, cause damage to mitochondrial proteins and lipids, promoting mitophagy. Although there are signs of accumulated oxidant stress in the SNc with aging and PD (53-55), it has not been proven that this damage originates from $\mathrm{Ca}^{2+}$-driven oxidative phosphorylation. If this were the case, basal rates of mitochondrial turnover or mitophagy should be high in SNc DA neurons and this rate should be diminished by inhibition of Cav1 $\mathrm{Ca}^{2+}$ channels. To test this hypothesis, a plasmid with a tyrosine hydroxylase $(\mathrm{TH})$ promoter driving an expression construct for the mitochondrially targeted variant of the coral protein Keima (mito-Keima) was packaged into an AAV and stereotaxically injected into the mesencephalon of mice. At physiological $\mathrm{pH}$, mito-Keima emits a photon more readily when stimulated with green light than red, but at acidic $\mathrm{pH}$, like that found in lysosomes, mito-Keima is best excited by red light (56, 57). Because Keima is poorly degraded in lysosomes, it provides a cumulative estimate of mitophagy over time. Thus, the ratio of mito-Keima fluorescence evoked by green to that evoked by red light provides a reliable measure of mitophagy in vivo (56). Three days after implantation with osmotic minipumps, mice were stereotaxically injected with $\mathrm{AAV}-\mathrm{TH}$-mito-Keima. Seven to ten days later, mice were sacrificed, ex vivo slices of the mesencephalon prepared, and mito-Keima imaged.

These experiments revealed 2 important features of mitophagy in SNc DA neurons. First, the basal rate of mitophagy in SNc DA neurons - as measured by the accumulation of mito-Keima in presumptive lysosomes - was significantly higher than in neighboring VTA DA neurons and in other basal ganglia neurons, such as globus pallidus externa ( $\mathrm{GPe}$ ) neurons, which spike at much higher rates than SNc DA neurons (Figure 5, D and F). Second, in SNc DA neurons from mice chronically treated with isradipine, the mitophagy rate was normalized, becoming similar to that in the other relatively PD-resistant neurons (Figure 5, E and F). These 2 observations provide direct support for the proposition that Cav1 channel-dependent oxidant stress in SNc DA neurons in vivo is sufficient to damage mitochondria and promote mitophagy. Moreover, these data show that chronic isradipine treatment diminishes mitochondrial oxidant stress enough in vivo to significantly lower mitophagy $(P<0.05)$.

Isradipine treatment elevated mitochondrial mass. Previous work based on electron microscopic (EM) analysis has suggested that mitochondrial density is unusually low in SNc DA neurons (58). To provide an alternative test of this inference, 3-color 2PLSM was used to optically section fixed slices of the mesencephalon from mice expressing mito-roGFP under control of the TH promoter. Sections were immunostained with a red antibody complex for TH (to delineate the cytoplasm) and with the blue DNA stain DAPI to mark the nucleus (Supplemental Figure 12). Optical sections were deconvolved, aligned, and assembled to construct a 3D map of the mitochondrial network in the soma and proximal dendrites of individual SNc and VTA DA neurons. This approach revealed that, as suggested previously, the proportion of cytoplasm occupied by mitochondria in SNc DA neurons was significantly smaller than that in neighboring VTA DA neurons and locus ceruleus (LC) neurons. (Figure 6, A and C). 
One possible explanation for the lower mitochondrial mass in SNC DA neurons is that their rate of mitophagy was higher. In principle, if the rate of mitochondrial biogenesis is fixed, then mitochondrial mass should be determined by the proportion of the total mitochondrial mass being turned over per unit time. If this were the case, then lowering the rate of mitophagy (or the proportion of mitochondria being degraded per unit time) with chronic isradipine treatment should increase mitochondrial mass in $\mathrm{SNc}$ DA neurons. Indeed, chronic (7 to 10 days) isradipine treatment did not change somatic volume (control, $n=14$; isradipine, $n=7$; Mann-Whitney $U$ test, $P=0.971$ ), but increased mitochondrial mass, bringing these values into the range found in VTA DA neurons (Figure 6, B and C). Treatment had no effect on mass in VTA DA neurons (Figure 6C). Thus, chronic isradipine treatment "normalized" mitochondrial density in SNc DA neurons.

Discontinuation of treatment reversed changes in mitochondrial density. A clinically (and biologically) important question is whether chronic isradipine treatment induces a lasting change in SNc DA neuron physiology. As noted above, removal of isradipine pumps led to a return of normal cytosolic $\left[\mathrm{Ca}^{2+}\right]$ during pacemaking. In principle then, the effects of isradipine treatment on mitochondrial biology should reverse in time. To test this hypothesis, mice were treated with isradipine-loaded osmotic minipumps for 7 to 10 days and then the pumps were removed. Three to four weeks after removal of the pumps, mice were sacrificed and mitochondrial mass in SNc DA neurons assessed as described above. Mass estimates returned to control values within this time frame (Figure 6C), arguing that the effects of isradipine treatment on mitochondrial biology were reversible.

\section{Discussion}

The studies presented yielded 4 observations. First, cytosolic $\left[\mathrm{Ca}^{2+}\right]$ during pacemaking was significantly higher in SNc DA neurons ex vivo than that in VTA neurons, rising from proximal to distal dendrites $(P<0.05)$. The oscillation in $\left[\mathrm{Ca}^{2+}\right]$, which reached high nanomolar levels in distal dendrites, was dependent in large measure upon the opening of Cav1 $\mathrm{Ca}^{2+}$ channels, particularly those with a Cav1.3 pore-forming subunit. Second, chronic inhibition of Cav1 channels in vivo with the negative allosteric modulator isradipine led to a sustained reduction in cytosolic $\left[\mathrm{Ca}^{2+}\right]$ in $\mathrm{SNc}$ DA neurons. This reduction was not accompanied by alterations in dopamine-dependent behavior, pacemaking rate, or the expression of Cav1 or Cav3 channel pore-forming subunit mRNAs. Third, chronic isradipine treatment significantly lowered somatodendritic mitochondrial oxidant stress, diminished a high basal rate of mitophagy, and increased mitochondrial mass in SNc DA neurons $(P<0.05)$. Fourth, with cessation of isradipine treatment, these effects reversed over the course of days. Together, these results fill an important gap in our understanding of mechanisms underlying the ongoing clinical trial with isradipine and how Cav1 channel inhibition might alter PD pathogenesis.

Somatodendritic $\mathrm{Ca}^{2+}$ oscillations were dependent upon Cav1 channels. Pacemaking in SNc DA neurons is accompanied by phase-locked oscillations in somatodendritic $\left[\mathrm{Ca}^{2+}\right](7,14,59,60)$. Using a proven strategy for measuring $\left[\mathrm{Ca}^{2+}\right]$ (33), our studies provide what we believe is the first quantitative estimate of the magnitude of these oscillations in ex vivo brain slices from young adult mice. Although $\mathrm{Ca}^{2+}$ buffering by Fura- 2 undoubtedly slowed and attenuated the cytosolic $\mathrm{Ca}^{2+}$ transient - leading to an underestimate of the normally occurring peaks in $\left[\mathrm{Ca}^{2+}\right]-$ this approach allowed meaningful comparisons to be made from cell to cell. In addition, because measurements were taken at a time point when the dye concentration had reached equilibrium, comparisons also could be made between subcellular regions. These comparisons revealed that during normal, slow pacemaking, average and peak $\left[\mathrm{Ca}^{2+}\right]$ estimates were higher in distal than proximal dendrites, rising into the high nanomolar range in distal dendrites during the spike. This proximal to distal gradient in dendritic $\left[\mathrm{Ca}^{2+}\right]$ is consistent with that seen in earlier work and attributable to the increasing surface area-to-volume ratio of dendrites as they taper (59).

As suggested by earlier work $(7,14,60)$, isradipine-sensitive Cav1 $\mathrm{Ca}^{2+}$ channels were found to be a major determinant of the oscillation in cytosolic $\left[\mathrm{Ca}^{2+}\right]$ during pacemaking. What has been less clear is the relative contribution of Cav1.2 and Cav1.3 $\mathrm{Ca}^{2+}$ channels to the oscillations, particularly in dendrites. The selective knockdown of low-threshold Cav1.3 channels significantly reduced the average and peak $\left[\mathrm{Ca}^{2+}\right]$ in dendrites as well as the ramp in $\left[\mathrm{Ca}^{2+}\right]$ prior to the spike, providing what we believe is the first clear demonstration that these channels are major contributors to subthreshold, dendritic $\mathrm{Ca}^{2+}$ dynamics (7-9). This observation, together with the fact that dendritic $\left[\mathrm{Ca}^{2+}\right]$ transients were largely unaffected by the failure of the perisomatic $\mathrm{Na}^{+}$spike, suggests that, although spikes can invade and entrain distal dendrites $(7,61)$, these regions are capable of autonomous membrane potential oscillations that are driven in large part by Cav1.3 channels $(7,8,59)$.

Given the privileged relationship between plasma membrane Cav1 channels and ryanodine receptors (RYRs) in other tissues $(62,63)$, the effect of isradipine on cytosolic $\left[\mathrm{Ca}^{2+}\right]$ is likely to reflect both the inhibition of plasma membrane Cav1 channels and the suppression of $\mathrm{Ca}^{2+}$-induced $\mathrm{Ca}^{2+}$ release (CICR) from the ER triggered by this influx $(13,64)$. This is important because CICR links the opening of plasma membrane Cav1 $\mathrm{Ca}^{2+}$ channels to mitochondrial $\mathrm{Ca}^{2+}$ loading and the stimulation of oxidative phosphorylation (65).

Because fluorescence imaging approaches spatially and temporal filter-intrinsic $\mathrm{Ca}^{2+}$ transients, our results suggest that dendritic $\left[\mathrm{Ca}^{2+}\right]$ rises into the low micromolar range during pacemaking. This is important because it means that 2 of the $\mathrm{Ca}^{2+}$-activated enzymes implicated in $\alpha$-synuclein aggregation and PD pathogenesis - calcineurin and calpain - should be persistently activated in SNc DA neuron dendrites, potentially driving atrophy and Lewy pathology (20, 36, 37, 66-69).

Chronic inhibition of Cav1 channels suppressed $\mathrm{Ca}^{2+}$ currents. In the ongoing phase 3 trial, target dosing of patients is $10 \mathrm{mg} / \mathrm{d}$ (70), given as $5 \mathrm{mg}$ instant release (IR) tablets (twice a day). Based upon the available pharmacokinetic data $(48,49), 5 \mathrm{mg}$ IR tablets should produce a peak plasma isradipine concentration of around $30 \mathrm{nM}$, declining to the 2 to $5 \mathrm{nM}$ range within 8 hours. Thus, our controlled release protocol, which was estimated to achieve plasma isradipine concentrations near $5 \mathrm{nM}$, should give us a comparable dosing. It is worth noting that our estimates are somewhat lower than those of 2 recent studies $(14.8 \mathrm{nM}$, ref. $32 ; 88.9 \mathrm{nM}$, ref. $47)$, but given the difficulties in estimating plasma isradipine concentration in rodents, the differences are probably not significant. 
The more translationally relevant question is whether low nanomolar plasma concentrations of isradipine are sufficient to produce a significant inhibition of Cav1 channels in the brain. This is a complicated question for several reasons. Although DHPs readily penetrate the blood-brain barrier (48), they are lipophilic and robustly accumulate in membrane, having partition coefficients greater than 5,000 (51). It is from within the membrane that they interact with Cav1 channels, as their DHP-binding site is on the external, lipid-interacting surface of the pore-forming subunit (71). This biology makes it critical that dose-response experiments be done at equilibrium. With this consideration in mind, our ex vivo brain slices were incubated with $10 \mathrm{nM}$ isradipine for 1 hour at near physiological temperatures. Isradipine at this concentration significantly diminished cytosolic $\mathrm{Ca}^{2+}$ oscillations in both proximal and distal dendrites of SNc DA neurons $(P<0.05)$. The magnitude of the reduction was consistent with that in previous dose-response experiments with Cav1.3 and Cav1.2 channels in heterologous systems (32) and other studies showing the contribution of Cav1 channels to the somatodendritic physiology of $\mathrm{SNc}$ DA neurons $(6-9,60)$. While in agreement with this literature, our results are at odds with a recent study that claimed $30 \mathrm{nM}$ isradipine had no effect on the somatic cytosolic $\left[\mathrm{Ca}^{2+}\right]$ in SNc DA neurons (32). However, the absence of appropriate positive controls and other issues, including uncertainty about whether measurements were taken at equilibrium and the failure to monitor dendritic $\left[\mathrm{Ca}^{2+}\right]$, make this negative result problematic.

The reduction in dendritic $\left[\mathrm{Ca}^{2+}\right]$ during pacemaking induced by acute application of $10 \mathrm{nM}$ isradipine was very similar to that seen in SNc DA neurons following chronic (7 to 10 days) treatment of mice. This reduction was not accompanied by any alteration in motor behavior or the expression of mRNAs for the pore-forming subunits of Cav1 or Cav3 $\mathrm{Ca}^{2+}$ channels. The effect of isradipine treatment was similar to that achieved by Cav1.3 subunit knockdown with an shRNA, particularly at subthreshold membrane potentials that preceded spike generation, suggesting that both Cav1.2 and Cav1.3 channels were significantly inhibited by peripheral drug administration $(P<0.05)$.

At first glance, the absence of an adaptation in spiking rate with isradipine treatment is puzzling. $\mathrm{Ca}^{2+}$ entry through Cav1.2 channels drives homeostatic control systems that maintain spike rate within predetermined bounds (72). Attenuating influx through these channels should, therefore, be interpreted as a slowing of spike rate, triggering a compensatory increase in rate. One possible explanation, although it seems unlikely, is that Cav1.2 channels are not used for homeostatic signaling in SNc DA neurons. Another possibility is that at the doses used, the inhibition achieved by isradipine was not primarily of Cav1.2 channels, but rather Cav1.3 channels, which are not used for homeostatic signaling. Why might Cav1.3 channels have been selectively inhibited? As mentioned above, isradipine, like other DHPs, is a voltagedependent-negative allosteric modulator. The affinity of DHPs increases with membrane depolarization, which presumably reflects alterations in the configuration of their binding pocket on the pore-forming subunit (71). It is plausible that this conformation change mirrors that of channel activation, which also depends upon a conformational change in the pore-forming subunit. If this is the case, then during pacemaking, Cav1.3 channels, which are activated at more hyperpolarized membrane potentials than Cav1.2 channels, should spend more time in the state leading to high-affinity DHP binding.

Chronic isradipine treatment lowered mitochondrial stress and turnover. Although systemic isradipine treatment did not change DA-dependent, open-field behavior, pacemaking rate, or $\mathrm{Ca}^{2+}$ channel expression, it did induce changes in mitochondria. As expected from previous studies using acute isradipine application $(13,14,73)$, chronic treatment lowered the high ambient level of mitochondrial oxidant stress in SNc DA neurons measured ex vivo. But was this the case in vivo? The most direct evidence in support of this proposition comes from the measurement of mitophagy rates. Mitochondrial oxidant stress can cause mitochondrial damage and, in principle at least, increase mitophagy (74). Indeed, there are clear signs of mitochondrial oxidant damage in the SNc of aged individuals and those with PD (75). However, there is no direct evidence that this damage is cell autonomous in origin, much less caused by Cav1 $\mathrm{Ca}^{2+}$ channels in otherwise healthy SNc DA neurons. Using virally delivered expression constructs for mito-Keima to track mitophagy in vivo $(56,57)$, it was found that mitophagy rates were high in SNc DA neurons compared with neighboring neurons and that these rates were normalized by systemic isradipine treatment. This is the first clear demonstration, to our knowledge, that in vivo mitochondrial oxidant stress driven by Cav1 $\mathrm{Ca}^{2+}$ channels is sufficient to damage mitochondria and increase mitophagy in healthy SNc DA neurons. Moreover, the ability of systemic isradipine at clinically relevant doses to lower stress and slow mitophagy not only provides a clear demonstration of meaningful target engagement in the brain, but also argues that this potential vulnerability of SNc DA neurons is therapeutically tractable.

This observation also provides an explanation for why mitochondrial mass is unusually low in SNc DA neurons - as judged both by a previous EM study (58) and our 2PLSM measurements. If mitochondrial biogenesis is relatively constant, then mitochondrial mass will be determined by the proportion of total mitochondria degraded per unit time. By lowering mitochondrial oxidant stress, isradipine appears to reduce this proportionality constant, increasing the steady-state mass of mitochondria in SNc DA neurons. However, while not the simplest explanation, it also is possible that isradipine treatment increases mitochondrial biogenesis to increase mass.

Translational implications. One of the goals of this study was to determine whether systemic administration of isradipine triggered compensatory adaptations in SNc DA neurons that would diminish its potential therapeutic benefit to PD patients. There were no deleterious adaptations observed in mice with chronic (7 to 10 days) isradipine treatment. This observation is consistent with the modest side-effect profile of chronic DHP use in humans (76). While it is possible that longer term treatment might induce unanticipated negative side effects, there were clearly beneficial effects of chronic isradipine treatment. Sustained lowering of $\mathrm{Ca}^{2+}$ entry through Cav1 channels diminished mitochondrial stress, damage, and turnover in vivo, normalizing mitochondrial mass in SNc DA neurons. This should lessen the impact of the mitochondrial challenges posed by advanced age, environmental toxins, and genetic mutations associated with PD (3). Chronic isradipine treatment also might alleviate proteostatic dysfunction associated with 
PD. One of the hallmarks of PD is Lewy pathology, the accumulation of intracellular aggregates of misfolded protein, including $\alpha$-synuclein. ROS and RNS, as well as high $\left[\mathrm{Ca}^{2+}\right]$, are known to promote $\alpha$-synuclein aggregation $(67,77-79)$; as isradipine treatment diminished all 3 aggregation triggers, it should slow $\alpha$-synuclein pathology. In addition, by reducing mitophagic load, isradipine should increase total autophagic capacity and the ability of SNc DA neurons to clear misfolded proteins and compensate for mutations in lysosomal enzymes, such as glucocerebrosidase $(80,81)$.

What is less clear from a translational standpoint is whether the initiation of isradipine treatment after motor symptom onset will be able to slow PD progression. At this point in the disease, there already is substantial loss of SNc DA neurons and it is unclear to what extent other factors, such as $\alpha$-synuclein pathology or inflammation, are driving pathogenesis $(82,83)$. The results of the ongoing phase 3 clinical trial that will be released in late 2018 will tell the tale. Moreover, in the ongoing clinical trial, isradipine is only being given at half of the daily FDA maximum dose because of concern over cardiovascular side effects (70). These side effects, such as peripheral edema, are attributable to inhibition of Cav1.2 channels. As Cav1.3 channels, rather than the more common Cav1.2 channels (84), appear to be the principal drivers of mitochondrial oxidant stress in SNc DA neurons, selective negative allosteric modulators could prove more effective neuroprotective agents than isradipine and have a narrower side-effect profile (44).

\section{Methods}

Animals. Male mice of ages P25-P35 and P5-P10 were used for these studies. Animals were housed and handled according to the guidelines established by the Northwestern University Animal Care and Use Committee, the NIH, and the Society for Neuroscience.

Tissue preparation. Mice were anesthetized with a ketamine/xylazine mixture, followed by a transcardial perfusion with ice-cold, oxygenated artificial cerebrospinal fluid (ACSF) containing $125 \mathrm{mM} \mathrm{NaCl}$, $2.5 \mathrm{mM} \mathrm{KCl}, 25 \mathrm{mM} \mathrm{NaHCO}_{3}, 1.25 \mathrm{mM} \mathrm{NaH}_{2} \mathrm{PO}_{4}, 2 \mathrm{mM} \mathrm{CaCl}_{2}, 1 \mathrm{mM}$ $\mathrm{MgCl}_{2}$, and $25 \mathrm{mM}$ glucose, $\mathrm{pH} 7.3$ (315-320 mOsm/l-1). After perfusion, mice were decapitated and brains removed rapidly, followed by sectioning in ice-cold oxygenated ACSF using a vibratome (VT1200S, Leica Microsystems). Coronal slices ( $220 \mu \mathrm{m}$ thick) were incubated in $\mathrm{ACSF}$ at $32-34^{\circ} \mathrm{C}$ for 30 minutes, then at room temperature for another 30 minutes before electrophysiological recordings. Slices were then transferred to a temperature-controllable Mini PTC chamber mounted on a 380-FM U Shifting Table (Luigs and Neumann). This system provided us with temperature control and manipulation/automation. Electrophysiological recordings were performed at $32-34^{\circ} \mathrm{C}$ unless specified otherwise. The chamber was superfused with carbogen-saturated ACSF. For pharmacological treatment, slices were preincubated for 60 minutes with a drug and then placed in a recording chamber with continuous superfusion of the same drug at the same concentration.

Electrophysiology/Ca ${ }^{2+}$ imaging. Conventional tight-seal (>2 G $\Omega$ ) whole-cell patch-clamp recordings were made on visually identified (LUMPLFL $\times 60$, 1.0 NA water-immersion objective, Dodt contrast system [Bruker Nano Fluorescence Microscopy] PC-e frame grabber card, and Micro-Manager software) ventral tier SNc neurons based on size, somatodendritic morphology, and regular spiking between 1 and $4 \mathrm{~Hz}$. Signals were filtered at $1-4 \mathrm{kHz}$ and digitized at $10 \mathrm{kHz}$ with a Prairie Ultima System running Prairie View 5.3 software (Bruker).
Neurons were dialyzed with a pipette solution containing $135 \mathrm{mM}$ $\mathrm{KMeSO}_{4}, 5 \mathrm{mM} \mathrm{KCl}, 5 \mathrm{mM}$ HEPES, $0.05 \mathrm{mM}$ EGTA, $10 \mathrm{mM}$ phosphocreatine-di(tris), $2 \mathrm{mM}$ ATP-Mg, and $0.5 \mathrm{mM}$ GTP-Na, the $\mathrm{pH}$ adjusted to 7.3 (290-300 mOsm/ $\left./ \mathrm{l}^{-1}\right)$. Perforated-patch experiments were performed with pipettes front filled with a solution containing $126 \mathrm{mM} \mathrm{KMeSO}_{4}, 14 \mathrm{mM} \mathrm{KCl}, 10 \mathrm{mM}$ HEPES, $1 \mathrm{mM} \mathrm{EGTA}, 0.5 \mathrm{mM}$ $\mathrm{CaCl}_{2}$, and $3 \mathrm{mM} \mathrm{MgCl}$ and then back filled with the same solution containing $20 \mathrm{mg} / \mathrm{ml}$ gramicidin-D. Cells were left to perforate until the spike height reached roughly $0 \mathrm{mV}$ before data collection began. Rapid jumps in the observed voltage to positive $(>0 \mathrm{mV})$ values were used as exclusion criteria due to break-in.

For $\mathrm{Ca}^{2+}$-imaging studies, EGTA was replaced with Fura-2 (100 $\mu \mathrm{M})$. $\mathrm{Ca}^{2+}$ imaging was performed with 2PLSM using a ChameleonUltra1 laser. Optical signals were acquired using 780-nm excitation (80 MHz pulse-repetition frequency and 250 fs pulse duration). Laser power attenuation was achieved with Pockels cell electrooptic modulators (model M350-80-02-BK, Conoptics) controlled by Prairie View. The laser-scanned images were acquired with a Prairie Ultima system. The fluorescence emission was collected by non-de-scanned photomultiplier tubes (PMTs). Fluorescence measurements were taken in a sample plane along dendritic segments $(10 \pm 5 \mu \mathrm{m}$ from the soma as proximal dendrites and $100 \pm$ $10 \mu \mathrm{m}$ from the soma as distal dendrites). Line-scan signals were acquired at 1 to $2 \mathrm{~ms}$ per line, 0.18 to $0.21 \mu \mathrm{m}$ pixel size, and 12 to $14 \mu$ s pixel dwell time for 6 to 9 seconds. $\left[\mathrm{Ca}^{2+}\right]$ was estimated using a single wavelength scheme (33). Using the fluorescence measurements as a function of time $(f[\mathrm{t}])$ generated by excitation at $780 \mathrm{~nm}$, $\left[\mathrm{Ca}^{2+}\right]$ was estimated using the following equation: $\left[\mathrm{Ca}^{2+}\right]=K_{d}[1-$ $\left.f(t) / f_{\text {max }}\right] /\left[f(t) / f_{\max }-1 / R_{f}\right](34,35)$. The 3 parameters of the equation $\left(K_{d}, R_{f}\right.$, and $\left.f_{\max }\right)$ were estimated as follows. The $K_{d}$ value used was $209 \mathrm{nM}$ to account for differences in experimental conditions. Previous work at $20^{\circ} \mathrm{C}, \mathrm{pH} 7.15, \mathrm{Ie}=0.1 \mathrm{M}$ and $0 \mathrm{mM} \mathrm{Mg}^{2+}$ estimated the Fura- $2 K_{d}$ to be $135 \mathrm{nM}$ (34). Our experiments were performed at $32^{\circ} \mathrm{C}, \mathrm{pH} 7.25, \mathrm{Ie}=0.185 \mathrm{M}$, and $2 \mathrm{mM} \mathrm{Mg}^{2+}$. Thus, the $K_{d}$ was adjusted based upon previous parametric work for $\mathrm{pH}$ (reduced by $21 \%$ ), ionic strength (increased by $86 \%$ ), $\mathrm{Mg}^{2+}$ concentration (increased by $8 \%$ ), and temperature (3\% reduction) (85). Using the same adjustments for Bis-fura, the $K_{d}$ was estimated to be 582 nM. $R_{f}$ was estimated to be 22 (33). The $f_{\max }$ value was obtained each time approximately 20 seconds prior to the measurements in current clamp by holding the cell at $-90 \mathrm{mV}$ to pull intracellular $\left[\mathrm{Ca}^{2+}\right]$ low. This strategy was validated using a conventional ratiometric approach: using 2 lasers set to 700 and $780 \mathrm{~nm}$, alternating images of the region of interest (ROI) in an SNc DA neuron in an ex vivo slice were obtained 25 to 30 minutes after beginning dialysis to allow dye concentration to equilibrate. Pixel size was $0.19 \mu \mathrm{m}$ and dwell time $12 \mu$ s; ROI size was between $80 \times 80 \mu \mathrm{m}$ and $125 \times 125$ $\mu \mathrm{m}$. Images were acquired for 20 seconds and repeated 3 times at different holding potentials (from -100 to -20 in $10 \mathrm{mv}$ steps) in regular ACSF. The same set of measurements was then obtained after superfusion with an ACSF lacking free $\mathrm{Ca}^{2+}$ and containing EGTA $(1.6 \mathrm{mM})$ and ionomycin $(1 \mu \mathrm{M})$. PicViewer (provided by $\mathrm{J}$. Dempster, Strathclyde University, Glasgow, United Kingdom) and IgorPro 6.22A (Wavemetrics) were used to analyze the data and compute the fluorescence ratio. An averaged ratio from 3 repeated images and 2 to 3 ROIs was generated and normalized to the ratio of $-90 \mathrm{mV}$ in $0 \mathrm{Ca}^{2+}$ ionomycin $\left(\mathrm{R} / \mathrm{R}_{\mathrm{I}-90 \mathrm{mV}}\right)$. An example is plotted 
in Supplemental Figure 1. These initial experiments consistently showed that at holding potentials below $-60 \mathrm{mV}$, the fluorescence ratio was stable and minimized; moreover, this ratio was indistinguishable from that obtained following ionomycin treatment and superfusion of the nominally $0 \mathrm{Ca}^{2+}$ solution. Thus, at negative membrane potentials below $60 \mathrm{mV}$, intracellular $\left[\mathrm{Ca}^{2+}\right]$ was below the detection limit for Fura-2. For single-wavelength estimation of $\left[\mathrm{Ca}^{2+}\right]$, at regular intervals during each experiment, the $f_{\max }$ value - operationally defined as the fluorescence obtained with $780 \mathrm{~nm}$ excitation at a holding potential of $-90 \mathrm{mV}$ - was obtained at the site of the line scan and used to estimate intracellular $\left[\mathrm{Ca}^{2+}\right]$ from fluorescence measurements $(f[t])$ during current clamp recordings. Current clamp recordings and imaging at each frequency were repeated at least 3 times. It is also important to note that at the time images were acquired, the kinetics of the spike-associated $\mathrm{Ca}^{2+}$ transient had stabilized, indicating that Fura-2 concentration had reached near its equilibrium value. For all fluorescence measurements, the background was subtracted.

Mitochondrial redox measurements. Transgenic mice expressing a mitochondrial matrix-targeted, redox-sensitive variant of GFP under control of the TH promoter (TH-mito-roGFP) were used for these experiments (14). Imaging and calibration of mito-roGFP fluorescence was performed as previously described (14).

Chronic isradipine treatment. Isradipine was delivered either by subcutaneous Alzet osmotic minipumps (models 1007D) or by pellets. Pumps were loaded with isradipine dissolved in vehicle $(50 \%$ DMSO/15\% PEG300/double-distilled water) at a concentration to achieve dosing of $3 \mu \mathrm{g} / \mathrm{g} / \mathrm{d}$. Prior to implantation, pumps were placed in $0.9 \%$ saline overnight at $37^{\circ} \mathrm{C}$ to insure immediate release of the drug. Pumps were implanted subcutaneously following the manufacturer's guidelines and left in place for 7 to 10 days. In a subset of experiments, 7 days after implantation, pumps were removed using sterile surgical procedures. For $\mathrm{Ca}^{2+}$ imaging, mice were sacrificed 5 to 7 days later. For relative mitochondrial mass measurement, mice were sacrificed 3 to 4 weeks later. Carrier-bound isradipine pellets (Innovative Research of America) with a $3 \mu \mathrm{g} / \mathrm{g} / \mathrm{d}$ daily dose were implanted subcutaneously. Plasma from both pellet- and pump-implanted mice was collected for liquid chromatography-tandem mass spectrometry (LC-MS/MS) analysis.

Knockdown of Cav1.3 mRNA. Target sequence (GGATCTCTCTCCCAAAGAA, position 248-270) from the nucleotide sequence of mouse Cav1.3 (accession no. NM_028981; NCBI's Nucleotide database) (46) was targeted by shRNAs to silence Cav1.3 gene expression in the SNc region. A Basic Local Alignment Research Tool (BLAST) (https://blast.ncbi.nlm.nih.gov/Blast.cgi) search of mouse genome databases confirmed that the target sequence was unique to the Cav1.3 transcript. Nontargeting control sequence AGGATCAAATTGATAGTAAACC (46) was used as a negative control. To construct shRNA vectors, 2 pairs of oligonucleotides containing the antisense sequence, hairpin loop region (TTCAAGAGA), and sense sequence with cohesive BamHI and XhoI sites were synthesized (Integrated DNA Technologies Inc.) and cloned into an adenoviral vector downstream of the U6 promoter. The vector also contained the red fluorescent protein (tdTomato) downstream of the CMV promoter. Plasmids were packaged into serotype $2 / 9 \mathrm{rAAVs}$; titers were roughly $2 \times 10^{13}$ viral genome copies/ml (Virovek). AAV9-U6-248-Cav1.3 shRNACMV-tdTomato-WPRE-bGHpA and AAV9-U6-control-shRNA-CMV-
tdTomato-WPRE-bGHpA AAVs were stereotaxically injected into the SNc of P21 mice at the volume of $200 \mathrm{nl}$ using the following stereotaxic coordinates: $3.5 \mathrm{~mm}$ posterior to bregma, $1.1 \mathrm{~mm}$ lateral to midline, and $4.2 \mathrm{~mm}$ deep from the cortical surface. Seven to ten days later, mice were sacrificed for electrophysiology and $\mathrm{Ca}^{2+}$ imaging. Infected neurons were identified by expression of tdTomato.

Real-time $q P C R$. For quantitation of Cav1.3 shRNA knockdown in the SNc tissue of rAAV-injected mice and changes of different LTCC transcript expressions after isradipine osmotic minipump treatment, brains were dissected and single $400 \mu \mathrm{m}$ sections of SNc were dissected and processed for RNA isolation using the RNeasy Mini Kit (QIAGEN). cDNA was synthesized from purified RNA using the SuperScript III Reverse Transcriptase Kit (Invitrogen). The relative abundance of different LTCC transcripts was measured by SYBR quantitative PCR using a $\triangle \mathrm{CT}$ method. All PCR reactions were done in duplicate. The following primers were used for PCR amplification of LTCC transcripts using SNc cDNA as a template. Mouse sequences were as follows: Cav1.3 (accession no. NM_028981): forward 5'-ACACCTTGATGTGGTCACTCTGCT-3', reverse 5'-TGTCCCATCACTGTTGAGAGGCAT-3; Cav1.2 (accession no. NM_009781): forward 5'-ACAGAAGGGAACCTAGAGCAAG-3', reverse 5'-GGATCAGGAAGGTGGCATAGAA-3'; Cav3.1 (accession no. NM_001112813): forward 5'-TTACCTGGGAGACACTTGGA-3, reverse 5'-CCAGCAGTAATGTGACGAGA-3'; Cav3.2 (accession no. XM_006524734.2): forward 5'-AGCACAGGAAAGCCAAGGTA-3', reverse 5'-TAGCTGTCATTGGGTAGCG-3'; Cav3.3 (accession no. XM_006520950.1): Cav3.3 forward 5'-ACACCCGAAACATCACCAAC-3', reverse 5'-CCACAGCAACAGCATCCAAT-3' (accession numbers are from NCBI's Nucleotide database). Mouse GAPDH (forward 5'-CATTTGCAGTGGCAAAGTGG-3', reverse 5'-GAATTTGCCGTGAGTGGAGT-3') and HPRT1 (forward 5'-GCTTGCTGGTGAAAAGGACCTCTCGAAG-3', reverse 5'CCCTGAAGTACTCATTATAGTCAAGGGCAT-3') were used to amplify GAPDH and HPRT1 CDNA as reference genes for the sample normalization. PCR reactions were performed with SYBR Green PCR Master Mix (Invitrogen) using the ABI StepOnePlus Real-Time PCR System (Applied Biosystems). The $\Delta C T$ values for each sample were calculated by subtracting the target CT value from the average CT value of the GAPDH and HPRT-1 reference genes $(\Delta \mathrm{CT}$ [sample] $=\mathrm{CT}$ targetv $-\mathrm{CT}[\mathrm{ref}])$, where target indicates target gene and ref indicates reference gene. The PCR products were verified by melt-curve analysis and agarose gels. The abundance of Cav3.3 transcripts was very low in SNc samples and thus not included in the isradipine qPCR data.

Mitophagy measurements. The mito-Keima plasmid was a gift from Toren Finkel (National Heart, Lung, and Blood Institute [NHLBI]/NIH). pFB-TH-SV40pA vector was created by replacing CMV promoter with PCR-amplified $2.5 \mathrm{~kb}$ rat $\mathrm{TH}$ promoter in $\mathrm{pFB}$ CMV-SV40pA vector (Virovek). pFB-TH-mito-Keima-SV4OpA vector was created by subcloning PCR-amplified mito-Keima into pFBTH-SV4OpA vector between EcoR1 and SalI sites. After sequence and expression verification, $\mathrm{pFB}-\mathrm{TH}-\mathrm{mt}-\mathrm{Keima}-\mathrm{SV} 4 \mathrm{OpA}$ vector was packaged into rAAVs using serotype 9 with $2.1 \times 10^{13}$ viral genome copies/ml (Virovek). AAV vector with human synapsin 1 promoter to drive mito-Keima expression was packaged with similar titer and serotype. To deliver vector, mice were anesthetized using the EZ Anesthesia System (Braintree Scientific) and immobilized on a stereotaxic frame (model $\mu 940$, David Kopf Instruments). Recom- 
binant AAV (400 nl) was injected with a glass micropipette (2-00000, Drummond Scientific Company) directly into the SNc region using the following stereotaxic coordinates: $3.3 \mathrm{~mm}$ posterior to bregma, $1 \mathrm{~mm}$ lateral to midline, and $4.2 \mathrm{~mm}$ deep from the cortical surface. AAV was injected into the GPe region using the following stereotaxic coordinates: $0.4 \mathrm{~mm}$ posterior to bregma, $1.8 \mathrm{~mm}$ lateral to midline, and $4 \mathrm{~mm}$ ventral from the bregma. Seven to ten days after injections, mice were sacrificed and slices were prepared as described above. All steps were performed at room temperature. Fluorescence of mito-Keima was imaged in 2 channels via 2 sequential excitations with Coherent lasers (480 nm, green; $561 \mathrm{~nm}$, red) controlled via PC and SlideBook 6 software (Intelligent Imaging Innovations). The emitted fluorescence signals were imaged after passing through a dual-band (488 and $561 \mathrm{~nm}$ ) emission filter (Semrock). Signals were acquired with $\mathrm{a} \times 60,1.0$ water-immersion objective, spinning-disk unit (Yokogawa), and an electron-multiplying charge-coupled device (CCD) (Photometrics). Image acquisition was achieved with SlideBook 6, and images were processed with no neighbor deconvolution using the same software. Image analysis was done using IMARIS 8.2 (Bitplane) and PicViewer provided by J. Dempster (Strathclyde University). The background subtraction and thresholding was done in Imaris. In a single plane of a neuron that contained the whole nucleus, 4 ROIs were selected in the cytoplasm and ratio was computed in PicViewer.

Mitochondrial mass measurement. Slices were prepared as described above and then fixed in $4 \%$ paraformaldehyde. After incubation with a monoclonal antibody to TH (1:1,000, Immunostar; ref. 86), bound primary antibodies were visualized by secondary antibodies coupled to Alexa Fluor 594 (Life Technologies). Cover glasses of $0.13 \mathrm{~mm}$ were used (VWR). Nuclear counterstain (NucBlue Fixed Cell Ready Probes Reagent, Thermo Fisher) was applied, and slices were mounted with Vectashield (Vector Labs). Images were acquired with a 2PLSM system (described above) using PlanApo 60×, 1.20 water-immersion lenses. Optical imaging of roGFP signals was acquired with $920 \mathrm{~nm}$ excitation with a pixel size of $0.132 \times 0.132 \mu \mathrm{m}$ and dwell time of 16 to $18 \mu \mathrm{s}$. The z step was $0.3 \mu \mathrm{m}$. Optical imaging of Alexa Fluor 594 and DAPI was acquired using $780 \mathrm{~nm}$ excitation with the same parameters as for roGFP. Each fluorescent signal was acquired sequentially. The roGFP and red and blue fluorescence were detected by a nondescanned GaAsP PMT (H7422PA, Hamamatsu). Image stacks of red, green, and blue signals were obtained by merging all signals with Imaris and deconvolved using AutoQuant 3 (Media Cybernetics) followed by $3 \mathrm{D}$ rendering and image analysis with Imaris software. Initial experiments (results not shown) were performed using antiGFP antibody to determine the overlap with TH-mito-roGFP. Relative mitochondrial mass and cytoplasm volume for each cell were determined using the Surface Object feature for which statistics was calculated in Imaris MeasurementPro. Representative images were processed with Adobe Photoshop CC (Adobe Systems Inc.) to adjust contrast and brightness.

Open-field behavior. Open-field tests were performed at the Northwestern University Behavioral Phenotyping Core. Animals were allowed to acclimate to the behavior room for at least 30 minutes before the test. For the test, the activity of each animal was recorded during a 5-minute exploration session in a $56 \times 56 \times 30 \mathrm{~cm}$ gray PVC open-field arena (Phenome Technologies Inc.) in a light- and sound- attenuating chamber (Industrial Acoustics Corp.). Red light background illumination was used to encourage movement and to allow the cameras to track the mice. Animals were gently placed in the center of the arena at the beginning of the test. Their movements were recorded with CCD cameras vertically mounted above and remotely controlled by the experimenter with Limelight software (Actimetrics). Arenas were cleaned between individual tests to prevent odor influences from the previous test. Tests were performed during the light phase of the circadian cycle, between 9 am and 5 pm. For analysis, each arena was divided into $25(5 \times 5)$ square grids. Total distance travelled $(\mathrm{cm})$ and time spent in each grid (\%) were analyzed with Actimetrics' Limelight software. Total time spent (\%) in 4 corner grids and 16 center grids was calculated.

Quantification of isradipine in the plasma using LC-MS/MS. Blood was drawn from anesthetized mice, incubated in tubes with $15 \% \mathrm{~K} 3$ EDTA solution for 30 minutes at room temperature, and then centrifuged at $10,000 \mathrm{~g}$ for 10 minutes at $4^{\circ} \mathrm{C}$. Collected supernatants were stored at $-80^{\circ} \mathrm{C}$ until further analysis. In one set of samples, isradipine plasma concentration was determined as previously described (19). Another set of samples was sent to Charles River Laboratories for LCMS/MS bioanalysis.

Statistics. Imaging data collected were analyzed with SlideBook, Imaris, Igor Pro 6.22A, or GraphPad Prism Version 6.0 (GraphPad Software). Summary data were displayed using box plots to avoid sampling distribution assumptions. Data are presented as box and whisker plots displaying median, lower, and upper quartiles, with whiskers representing minimum and maximum of the data. Differences between samples were evaluated using nonparametric Mann-Whitney $U$ test with Dunn's correction for multiple comparisons. A $P$ value of less than 0.05 was considered significant. Statistical analysis was performed with GraphPad Prism Version 6.0.

Study approval. All animal protocols were approved by the IACUC at Northwestern University.

\section{Author contributions}

JNG, EI, PTS, and DJS conceived and designed the project. JNG, EI, BY, JSP, and DG executed the stereotaxic injection and electrophysiological and $\mathrm{Ca}^{2+}$ imaging experiments, performed data analysis, and prepared figures. DW provided feedback on the imaging strategy and the execution of the imaging experiments. JK redesigned and tested the Cav1.3 shRNAs and executed the knockdown and qPCR experiments. JNG, EI, BY, and DJS composed the figures. DJS, JNG, PTS, BY, and EI wrote the manuscript.

\section{Acknowledgments}

This work was supported by awards to DJS by the JPB Foundation, the IDP Foundation, and the NIH (NS NS047085). We wish to thank Toren Finkel for providing the mito-Keima plasmid, Savio Chan and Harry Xenias for guidance and technical assistance with the Coherent laser spinning-disk unit, and Craig Weiss, Mary Kando, and the Northwestern University Behavioral Phenotyping Core for help with open-field tests and data analysis.

Address correspondence to: D. James Surmeier, Department of Physiology, Feinberg School of Medicine, Northwestern University, 303 E. Chicago Avenue, Chicago, Illinois 60611 USA. Phone: 312.503.4904; Email: j-surmeier@northwestern.edu. 
1. Hornykiewicz O. Dopamine (3-hydroxytyramine) and brain function. Pharmacol Rev. 1966;18(2):925-964.

2. Berg D, et al. Time to redefine PD? Introductory statement of the MDS Task Force on the definition of Parkinson's disease. Mov Disord. 2014;29(4):454-462.

3. Surmeier DJ, Obeso JA, Halliday GM. Selective neuronal vulnerability in Parkinson disease. Nat Rev Neurosci. 2017;18(2):101-113.

4. Grace AA, Bunney BS. The control of firing pattern in nigral dopamine neurons: single spike firing. J Neurosci. 1984;4(11):2866-2876.

5. Hyland BI, Reynolds JN, Hay J, Perk CG, Miller R. Firing modes of midbrain dopamine cells in the freely moving rat. Neuroscience. 2002;114(2):475-492.

6. Nedergaard S, Flatman JA, Engberg I. Nifedipine- and omega-conotoxin-sensitive $\mathrm{Ca} 2+$ conductances in guinea-pig substantia nigra pars compacta neurones. J Physiol (Lond). 1993;466:727-747.

7. Guzman JN, Sánchez-Padilla J, Chan CS, Surmeier DJ. Robust pacemaking in substantia nigra dopaminergic neurons. J Neurosci. 2009;29(35):11011-11019.

8. Puopolo M, Raviola E, Bean BP. Roles of subthreshold calcium current and sodium current in spontaneous firing of mouse midbrain dopamine neurons. J Neurosci. 2007;27(3):645-656.

9. Putzier I, Kullmann PH, Horn JP, Levitan ES. Cav1.3 channel voltage dependence, not $\mathrm{Ca} 2+$ selectivity, drives pacemaker activity and amplifies bursts in nigral dopamine neurons. J Neurosci. 2009;29(49):15414-15419.

10. Kann O, Kovács R. Mitochondria and neuronal activity. Am J Physiol, Cell Physiol. 2007;292(2):C641-C657.

11. Llorente-Folch I, Rueda CB, Pardo B, Szabadkai G, Duchen MR, Satrustegui J. The regulation of neuronal mitochondrial metabolism by calcium. JPhysiol (Lond). 2015;593(16):3447-3462.

12. Glancy B, Balaban RS. Role of mitochondrial $\mathrm{Ca} 2+$ in the regulation of cellular energetics. Biochemistry. 2012;51(14):2959-2973.

13. Sanchez-Padilla J, et al. Mitochondrial oxidant stress in locus coeruleus is regulated by activity and nitric oxide synthase. Nat Neurosci. 2014;17(6):832-840.

14. Guzman JN, et al. Oxidant stress evoked by pacemaking in dopaminergic neurons is attenuated by DJ-1. Nature. 2010;468(7324):696-700.

15. Dragicevic E, Schiemann J, Liss B. Dopamine midbrain neurons in health and Parkinson's disease: emerging roles of voltage-gated calcium channels and ATP-sensitive potassium channels. Neuroscience. 2015;284:798-814.

16. Schapira AH, Olanow CW, Greenamyre JT, Bezard E. Slowing of neurodegeneration in Parkinson's disease and Huntington's disease: future therapeutic perspectives. Lancet. 2014;384(9942):545-555.

17. Requejo-Aguilar R, Bolaños JP. Mitochondrial control of cell bioenergetics in Parkinson's disease. Free Radic Biol Med. 2016;100:123-137.

18. Klein C, Krainc D. Glucocerebrosidase mutations: tipping point toward Parkinson disease and dementia? JAMA Neurol. 2013;70(6):686-688.
19. Ilijic E, Guzman JN, Surmeier DJ. The L-type channel antagonist isradipine is neuroprotective in a mouse model of Parkinson's disease. Neurobiol Dis. 2011;43(2):364-371.

20. Chan CS, et al. 'Rejuvenation' protects neurons in mouse models of Parkinson's disease. Nature. 2007;447(7148):1081-1086.

21. Kupsch A, Sautter J, Schwarz J, Riederer P, Gerlach M, Oertel WH. 1-Methyl-4-phenyl-1,2,3,6tetrahydropyridine-induced neurotoxicity in non-human primates is antagonized by pretreatment with nimodipine at the nigral, but not at the striatal level. Brain Res. 1996;741(1-2):185-196.

22. Singh A, Verma P, Balaji G, Samantaray S, Mohanakumar KP. Nimodipine, an L-type calcium channel blocker attenuates mitochondrial dysfunctions to protect against 1-methyl-4-phenyl-1,2,3,6-tetrahydropyridine-induced Parkinsonism in mice. Neurochem Int. 2016;99:221-232.

23. Lee YC, Lin CH, Wu RM, Lin JW, Chang CH, Lai MS. Antihypertensive agents and risk of Parkinson's disease: a nationwide cohort study. PLoS One. 2014;9(6):e98961.

24. Pasternak B, Svanström H, Nielsen NM, Fugger L, Melbye M, Hviid A. Use of calcium channel blockers and Parkinson's disease. Am JEpidemiol. 2012;175(7):627-635.

25. Marras C, et al. Dihydropyridine calcium channe blockers and the progression of parkinsonism. Ann Neurol. 2012;71(3):362-369.

26. Ritz B, Rhodes SL, Qian L, Schernhammer E, Olsen JH, Friis S. L-type calcium channel blockers and Parkinson disease in Denmark. Ann Neurol. 2010;67(5):600-606.

27. Becker C, Jick SS, Meier CR. Use of antihypertensives and the risk of Parkinson disease. Neurology. 2008;70(16 Pt 2):1438-1444.

28. Gudala K, Kanukula R, Bansal D. Reduced risk of parkinson's disease in users of calcium channel blockers: a meta-analysis. Int J Chronic Dis. 2015;2015:697404.

29. Biglan KM, et al. A novel design of a phase III trial of isradipine in early Parkinson disease (STEADY-PD III). Ann Clin Transl Neurol. 2017;4(6):360-368

30. Surmeier DJ, Guzman JN, Sánchez-Padilla J. Calcium, cellular aging, and selective neuronal vulnerability in Parkinson's disease. Cell Calcium. 2010;47(2):175-182.

31. Poetschke C, et al. Compensatory T-type $\mathrm{Ca} 2+$ channel activity alters D2-autoreceptor responses of Substantia nigra dopamine neurons from Cav1.3 L-type Ca2+ channel KO mice. Sci Rep. 2015;5:13688.

32. Ortner NJ, et al. Lower affinity of isradipine for L-type $\mathrm{Ca} 2+$ Channels during substantia nigra dopamine neuron-like activity: implications for neuroprotection in Parkinson's disease. J Neurosci. 2017;37(28):6761-6777.

33. Wokosin DL, Loughrey CM, Smith GL. Characterization of a range of fura dyes with two-photon excitation. Biophys J. 2004;86(3):1726-1738.

34. Grynkiewicz G, Poenie M, Tsien RY. A new generation of $\mathrm{Ca} 2+$ indicators with greatly improved fluorescence properties. J Biol Chem. 1985;260(6):3440-3450.

35. Maravall M, Mainen ZF, Sabatini BL, Svoboda K. Estimating intracellular calcium concentrations and buffering without wavelength ratioing. BiophysJ. 2000;78(5):2655-2667.

36. Patt S, Gertz HJ, Gerhard L, Cervós-Navarro J. Pathological changes in dendrites of substantia nigra neurons in Parkinson's disease: a Golgi study. Histol Histopathol. 1991;6(3):373-380.

37. Cruz-Sánchez FF, Cardozo A, Tolosa E. Neuronal changes in the substantia nigra with aging: a Golgi study. J Neuropathol Exp Neurol. 1995;54(1):74-81.

38. Hage TA, Sun Y, Khaliq ZM. Electrical and $\mathrm{Ca}(2+)$ signaling in dendritic spines of substantia nigra dopaminergic neurons. Elife. 2016;5:13905.

39. Roeper J. Dissecting the diversity of midbrain dopamine neurons. Trends Neurosci. 2013;36(6):336-342.

40. Wolfart J, Roeper J. Selective coupling of T-type calcium channels to SK potassium channels prevents intrinsic bursting in dopaminergic midbrain neurons. J Neurosci. 2002;22(9):3404-3413.

41. Dragicevic E, et al. Cav1.3 channels control D2-autoreceptor responses via NCS-1 in substantia nigra dopamine neurons. Brain. 2014;137(Pt 8):2287-2302.

42. Shipe WD, et al. Design, synthesis, and evaluation of a novel 4-aminomethyl-4-fluoropiperidine as a T-type $\mathrm{Ca} 2+$ channel antagonist. J Med Chem. 2008;51(13):3692-3695.

43. Dreyfus FM, et al. Selective T-type calcium channel block in thalamic neurons reveals channel redundancy and physiological impact of $\mathrm{I}(\mathrm{T})$ window. J Neurosci. 2010;30(1):99-109.

44. Kang S, et al. CaV1.3-selective L-type calcium channel antagonists as potential new therapeutics for Parkinson's disease. Nat Commun. 2012;3:1146.

45. Huang H, Ng CY, Yu D, Zhai J, Lam Y, Soong TW. Modest CaV1.342-selective inhibition by compound 8 is $\beta$-subunit dependent. Nat Commun . 2014;5:4481.

46. Schierberl K, et al. Cav1.2 L-type Ca2+ channels mediate cocaine-induced GluA1 trafficking in the nucleus accumbens, a long-term adaptation dependent on ventral tegmental area Cav1.3. Channels (Austin). 2012; 6(1):11-17.

47. Anekonda TS, Quinn JF, Harris C, Frahler K, Wadsworth TL, Woltjer RL. L-type voltage-gated calcium channel blockade with isradipine as a therapeutic strategy for Alzheimer's disease. Neurobiol Dis. 2011;41(1):62-70.

48. Johnson BA, et al. Kinetic and cardiovascular comparison of immediate-release isradipine and sustained-release isradipine among nontreatment-seeking, cocaine-dependent individuals. Prog Neuropsychopharmacol Biol Psychiatry. 2005;29(1):15-20.

49. Park JH, et al. Quantification of isradipine in human plasma using LC-MS/MS for pharmacokinetic and bioequivalence study. JChromatogr B Analyt Technol Biomed Life Sci. 2009;877(1-2):59-64.

50. Herbette LG, Vant Erve YM, Rhodes DG. Interaction of 1,4 dihydropyridine calcium channel antagonists with biological membranes: lipid bilayer partitioning could occur before drug binding to receptors. J Mol Cell Cardiol. 1989;21(2):187-201.

51. Bean BP. Nitrendipine block of cardiac calcium channels: high-affinity binding to the 
inactivated state. Proc Natl Acad Sci U S A. 1984;81(20):6388-6392.

52. Surmeier DJ, Schumacker PT, Guzman JD, Ilijic E, Yang B, Zampese E. Calcium and Parkinson's disease. Biochem Biophys Res Commun. 2017;483(4):1013-1019.

53. Reeve AK, et al. Nature of mitochondrial DNA deletions in substantia nigra neurons. Am J Hum Genet. 2008;82(1):228-235.

54. Bender A, et al. High levels of mitochondrial DNA deletions in substantia nigra neurons in aging and Parkinson disease. Nat Genet. 2006;38(5):515-517.

55. Kraytsberg Y, Kudryavtseva E, McKee AC, Geula C, Kowall NW, Khrapko K. Mitochondrial DNA deletions are abundant and cause functional impairment in aged human substantia nigra neurons. Nat Genet. 2006;38(5):518-520.

56 . Sun N, et al. Measuring in vivo mitophagy. Mol Cell. 2015;60(4):685-696.

57. Katayama H, Kogure T, Mizushima N, Yoshimori T, Miyawaki A. A sensitive and quantitative technique for detecting autophagic events based on lysosomal delivery. Chem Biol. 2011;18(8):1042-1052.

58. Liang CL, Wang TT, Luby-Phelps K, German DC. Mitochondria mass is low in mouse substantia nigra dopamine neurons: implications for Parkinson's disease. Exp Neurol. 2007;203(2):370-380.

59. Wilson CJ, Callaway JC. Coupled oscillator model of the dopaminergic neuron of the substantia nigra. J Neurophysiol. 2000;83(5):3084-3100.

60 . Hage TA, Khaliq ZM. Tonic firing rate controls dendritic $\mathrm{Ca} 2+$ signaling and synaptic gain in substantia nigra dopamine neurons. JNeurosci. 2015;35(14):5823-5836.

61. Häusser M, Stuart G, Racca C, Sakmann B. Axonal initiation and active dendritic propagation of action potentials in substantia nigra neurons. Neuron. 1995;15(3):637-647.

62. Fill M, Copello JA. Ryanodine receptor calcium release channels. Physiol Rev. 2002;82(4):893-922.

63. Lanner JT, Georgiou DK, Joshi AD, Hamilton SL. Ryanodine receptors: structure, expres- sion, molecular details, and function in calcium release. Cold Spring Harb Perspect Biol. 2010;2(11):a003996.

64. Morikawa H, Khodakhah K, Williams JT. Two intracellular pathways mediate metabotropic glutamate receptor-induced Ca2+ mobilization in dopamine neurons. JNeurosci. 2003;23(1):149-157.

65. Rizzuto R, Pozzan T. Microdomains of intracellular Ca2+: molecular determinants and functional consequences. Physiol Rev. 2006;86(1):369-408.

66. Caraveo G, et al. Calcineurin determines toxic versus beneficial responses to $\alpha$-synuclein. Proc Natl Acad Sci U S A. 2014;111(34):E3544-E3552.

67. Diepenbroek M, et al. Overexpression of the calpain-specific inhibitor calpastatin reduces human alpha-Synuclein processing, aggregation and synaptic impairment in [A3OP] $\alpha$ Syn transgenic mice. Hum Mol Genet. 2014;23(15):3975-3989.

68. Goll DE, Thompson VF, Li H, Wei W, Cong J. The calpain system. Physiol Rev. 2003;83(3):731-801.

69. Stewart AA, Ingebritsen TS, Manalan A, Klee CB, Cohen P. Discovery of a Ca2+- and calmodulindependent protein phosphatase: probable identity with calcineurin (CaM-BP80). FEBS Lett. 1982;137(1):80-84.

70. Surmeier DJ, Halliday GM, Simuni T. Calcium, mitochondrial dysfunction and slowing the progression of Parkinson's disease. Exp Neurol. 2017;298(Pt B):202-209.

71. Tang L, et al. Structural basis for inhibition of a voltage-gated $\mathrm{Ca} 2+$ channel by $\mathrm{Ca} 2+$ antagonist drugs. Nature. 2016;537(7618):117-121.

72. Turrigiano GG. Homeostatic plasticity in neuronal networks: the more things change, the more they stay the same. Trends Neurosit. 1999;22(5):221-227.

73. Goldberg JA, et al. Calcium entry induces mitochondrial oxidant stress in vagal neurons at risk in Parkinson's disease. Nat Neurosci. 2012;15(10):1414-1421.

74. Sun N, Youle RJ, Finkel T. The Mitochondrial Basis of Aging. Mol Cell. 2016;61(5):654-666.

75. Reeve A, Simcox E, Turnbull D. Ageing and Par- kinson's disease: why is advancing age the biggest risk factor? Ageing Res Rev. 2014;14:19-30.

76. Epstein BJ, Vogel K, Palmer BF. Dihydropyridine calcium channel antagonists in the management of hypertension. Drugs. 2007;67(9):1309-1327.

77. Mullin S, Schapira A. $\alpha$-Synuclein and mitochondrial dysfunction in Parkinson's disease. Mol Neurobiol. 2013;47(2):587-597.

78. Rcom-H'cheo-Gauthier AN, Davis A, Meedeniya ACB, Pountney DL. Alpha-synuclein aggregates are excluded from calbindin-D28k-positive neurons in dementia with Lewy bodies and a unilateral rotenone mouse model. Mol Cell Neurosi. 2016;77:65-75.

79. Rcom-H'cheo-Gauthier A, Goodwin J, Pountney DL. Interactions between calcium and alphasynuclein in neurodegeneration. Biomolecules. 2014;4(3):795-811.

80. Gegg ME, Schapira AH. Mitochondrial dysfunction associated with glucocerebrosidase deficiency. Neurobiol Dis. 2016;90:43-50.

81. Krainc D. Clearance of mutant proteins as a therapeutic target in neurodegenerative diseases. Arch Neurol. 2010;67(4):388-392.

82. Wong YC, Krainc D. $\alpha$-synuclein toxicity in neurodegeneration: mechanism and therapeutic strategies. Nat Med. 2017;23(2):1-13.

83. Subramaniam SR, Federoff HJ. Targeting microglial activation states as a therapeutic avenue in Parkinson's disease. Front Aging Neurosci. 2017;9:176.

84. Sinnegger-Brauns MJ, et al. Expression and 1,4-dihydropyridine-binding properties of brain L-type calcium channel isoforms. Mol Pharmacol. 2009;75(2):407-414.

85. Harrison SM, Bers DM. The effect of temperature and ionic strength on the apparent Ca-affinity of EGTA and the analogous Ca-chelators BAPTA and dibromo-BAPTA. Biochim Biophys Acta. 1987;925(2):133-143.

86. Chan P, Di Monte DA, Langston JW, Janson AM. (+)MK-801 does not prevent MPTP-induced loss of nigral neurons in mice. J Pharmacol Exp Ther. 1997;280(1):439-446. 